\title{
Re-annotation of eight Drosophila genomes
}

\author{
Haiwang Yang ${ }^{1} \mathbb{D}$, Maria Jaime ${ }^{1}$, Maxi Polihronakis², Kelvin Kanegawa ${ }^{3}$, Therese Markow ${ }^{4,2}$, Kenneth Kaneshiro ${ }^{3}$, \\ Brian Oliver ${ }^{1}$
}

\begin{abstract}
The sequenced genomes of the Drosophila phylogeny are a central resource for comparative work supporting the understanding of the Drosophila melanogaster non-mammalian model system. These have also facilitated evolutionary studies on the selected and random differences that distinguish the thousands of extant species of Drosophila. However, full utility has been hampered by uneven genome annotation. We have generated a large expression profile dataset for nine species of Drosophila and trained a transcriptome assembly approach on $D$. melanogaster that best matched the extensively curated annotation. We then applied this to the other species to add more than 10000 transcript models per species. We also developed new orthologs to facilitate cross-species comparisons. We validated the new annotation of the distantly related Drosophila grimshawi with an extensive collection of newly sequenced CDNAs. This reannotation will facilitate understanding both the core commonalities and the species differences in this important group of model organisms, and suggests a strategy for annotating the many forthcoming genomes covering the tree of life.
\end{abstract}

DOI 10.26508/lsa.201800156 | Received 13 August 2018 | Revised 15 December 2018 | Accepted 16 December 2018 | Published online 24 December 2018

\section{Background}

Drosophila melanogaster is a genetic and genomic workhorse that has led to the understanding of the chromosome theory of inheritance, the nature of mutations, pattern formation in development, innate immunity, circadian rhythms, and a host of other discoveries in the last century (Bilder \& Irvine, 2017; Callaway \& Ledford, 2017). There is a core set of 12 sequenced and assembled genomes in the Drosophila genus (Adams et al, 2000; Richards et al, 2005; Drosophila 12 Genomes Consortium et al, 2007; Hoskins et al, 2015). This is an important resource for studying diverse evolutionary biology problems, such as sex chromosome evolution (Charlesworth \& Charlesworth, 2005), de novo gene formation (Lu et al, 2008), and duplication and divergence (Vieira et al, 2007). Using other Drosophila species for comparative genomics can also help identify the conserved genomic elements in D. melanogaster, in cases where frequent random occurrence obscures identification of DNA elements (Chen et al, 2014). For example, comparative genomics was a valuable tool for studying Doublesex DNA binding site function, as the short degenerate sequences bound by Doublesex appear by chance at a high rate (Clough et al, 2014). Comparative genomics is also essential for determining the probable function of transcribed elements. For example, short ORFs in "noncoding" RNAs are not commonly annotated because they occur often in a random sequence. But if a short ORF appears in a phylogeny, then those "noncoding" RNAs are likely to encode short biologically active peptides (Tautz, 2009).

The utility of the sequenced and annotated Drosophila genomes is clear, but there is room for improvement. The current annotations of non-melanogaster members of the genus are uneven and inferior to the heavily and actively curated D. melanogaster annotation (Gramates et al, 2017). For example, although there have been six versions of the $D$. melanogaster genome and upwards of 75 annotations (Hoskins et al, 2015), most the other species have a single assembly and one or two annotation versions (Richards et al, 2005; Drosophila 12 Genomes Consortium et al, 2007; Hu et al, 2013). Much of genome annotation depends on the identification of conserved long ORFs, but expression data presents a direct way to determine what portions of the genome are actively transcribed and should be annotated. There are transcript-driven annotation tools, such as Gnomon (Souvorov et al, 2010), but these are generic and not tuned to a particular lineage.

Dissected adult tissues are a good source for mRNAs to support genome annotation (Chintapalli et al, 2007; Chen et al, 2014). Although most tissues are present in both sexes, there are some sexspecific organs that show unique expression profiles (Arbeitman et al, 2002; Parisi et al, 2004; Graveley et al, 2011; Brown et al, 2014). For example, there are approximately 8,000 genes preferentially expressed in the testis and male reproductive tract and $\sim 5,000$ genes preferentially expressed in the ovary and female reproductive tract. In addition, because female transcripts maternally deposited in eggs are used during embryogenesis, many developmentally important transcripts are detected in adult female ovary samples. Overall, using dissected tissues from adults increases

\footnotetext{
${ }^{1}$ Section of Developmental Genomics, Laboratory of Cellular and Developmental Biology, National Institute of Diabetes and Digestive and Kidney Diseases, National Institutes of Health, Bethesda, MD, USA ${ }^{2}$ Drosophila Species Stock Center, Division of Biological Sciences, University of California San Diego, La Jolla, CA, USA ${ }^{3}$ Hawaiian Drosophila Research Stock Center, Pacific Biosciences Research Center, University of Hawai'i at Manoa, Honolulu, HI, USA ${ }^{4}$ National Laboratory of Genomics for Biodiversity (LANGEBIO), Irapuato, Guanajuato, Mexico
}

Correspondence: haiwangyang@gmail.com 
coverage compared with whole samples, because of the fact that genes rarely expressed in a whole organism often show enriched expression in a given tissue (Chintapalli et al, 2007). More than $85 \%$ of annotated genes are expected to be covered in such experiments (Chintapalli et al, 2007; Daines et al, 2011). Although a full developmental profile, use of multiple genetic backgrounds, and environmental and/or genetic perturbations might marginally increase the coverage, these are less cost-effective than using adult tissues. In this work, we have used Poly- $A^{+}$RNA-seq expression profiling of 584 samples from adults of D. melanogaster (Dmel), Drosophila yakuba (Dyak), Drosophila ananassae (Dana), Drosophila pseudoobscura (Dpse), Drosophila persimilis (Dper), Drosophila willistoni (Dwil), Drosophila mojavensis (Dmoj), Drosophila virilis (Dvir), and Drosophila grimshawi (Dgri) to support reannotation of the corresponding genomes.

Performing de novo annotation based on gene expression is complicated by RNA coverage gaps that result in discontinuity within a single transcription unit, overlapping genes, and false splice junction calls due to gap generation that maximizes read alignment (Robertson et al, 2010; Sturgill et al, 2013). As a result, reference annotations and de novo transcript assemblies can differ radically (Garber et al, 2011; Haas et al, 2013). Some of these difficulties can be overcome, for example by using methods that capture strandedness (Grabherr et al, 2011). In addition, tuning the transcript parameters can improve the quality of the transcriptome (Vijay et al, 2013). These tools are often run using default parameters or based on some simple assumptions and tests. In this work, we decided to systematically test parameters and train support vector machines (SVM) on D. melanogaster, and then lift over these settings for automated annotation of the remaining species. This resulted in dramatic improvements in the mapping of RNA-seq reads to a greatly expanded set of genes and isoforms in these species. This general method might also be broadly applicable. If a few select species in the tree of life are targeted for heavily manual annotation, this can inform the automated annotation of the entire phylogeny.

\section{Results}

\section{RNA-seq}

Dmel, Dyak, Dana, Dpse, Dper, Dwil, Dmoj, Dvir, and Dgri represent a wide range of species separated by an estimated 40 million years of evolution (Leung et al, 2015), with fully saturated neutral substitutions at the widest separations (Fig 1A; after [Chen et al, 2014]). We targeted these genomes for re-annotation. We also included two Dmel strains: $W^{1118}$ and Oregon-R (OreR) to facilitate training the annotation. To evaluate the annotations of these nine members of the genus, we performed stranded Poly- $\mathrm{A}^{+} \mathrm{RNA}$-seq experiments on quadruplicate biological samples derived from sexed whole flies and tissues (covering up to eight adult tissue types for each sex: whole organisms, gonads, reproductive tracts, terminalia, thoraxes, viscera, heads, and abdomens) for a total of 584 samples and $\sim 5$ billion RNA-seq reads (available at the Gene Expression Omnibus (GEO) [Edgar et al, 2002], under accession GSE99574 and a subset of accession GSE80124, see the Materials and Methods section).

\section{Annotation evaluation and optimized re-annotation}

We first used RNA-seq data to evaluate the existing annotations. If an annotation was complete, the vast majority of our RNA-seq reads would map to annotated transcripts. On the other hand, if an annotation was poor, we would observe more RNA-seq reads mapping to unannotated regions and there might be extensive unannotated regions with mapped reads (Fig 1B). To determine how many reads aligning to the genomes mapped to annotated genes and transcripts, and how well those reads covered the existing models, we calculated the ratio of reads uniquely mapped to unannotated regions relative to the ones uniquely mapped to the whole genome by tissue type and species (Fig 1C). This metric was sensitive to read abundance from highly expressed unannotated genes. Therefore, in addition to the number of RNA-seq reads, we used a related metric describing the number of bases covered by at least one RNA-seq read outside of the annotations (Fig 1D).

We observed a wide range of annotation qualities using these simple metrics. For example, Dmel had only $1 \%$ of reads uniquely mapped to unannotated regions for all tissues except male reproductive tract, which had $2 \%$ mapping to unannotated regions, strongly suggesting that the Dmel genome annotation for highly expressed genes is nearly complete (Fig 1C). However, we still observed read alignment at up to $14 \%$ of unannotated Dmel regions (Fig 1D), suggesting that additional annotation is required, especially for fully capturing the transcriptomes of the testis, head, and thorax. In contrast, we observed that up to $46 \%$ of reads are uniquely mapped to unannotated regions in non-melanogaster species (Fig 1C). Similarly, we observed that up to $40 \%$ of unannotated regions had mapped read coverage (Fig 1D). Dper and Dgri had the poorest annotations. To determine if certain tissues might be especially valuable in completing the transcriptome, we also examined the reads mapping ratios by tissue. As we observed for Dmel, we found that RNAs from the ovary, reproductive tract, thorax, viscera, and abdomen had the best mapping, whereas RNAs from the testis and head of either sex had the poorest mapping to the annotations. In conclusion, our results suggested that all eight of the non-melanogaster annotations need major improvements to approach the quality of the Dmel annotation.

Given the clear superiority of the Dmel annotations, we decided to systematically develop de novo transcriptome annotations for the genus that would approximate the Dmel annotation quality. To determine the best method for generating these new annotations, we generated tens of thousands of de novo annotations of Dmel, where we systematically and iteratively honed-in on optimal transcript assembly algorithm parameters (nine StringTie parameters and $>1 / 4$ million combinations), and used SVMs to develop filtering criteria such that we most closely matched the Dmel standard. We then used these settings and filters to generate new annotations for the remaining species (see the Materials and Methods section). We were gratified to find that in non-melanogaster species, the reads mapping to unannotated regions decreased fivefold after the annotation update (Fig $1 \mathrm{E}$ and F; number of reads in species and tissues before and after annotation update: Wilcoxon rank test, $\left.P<2.2 \times 10^{-16}\right)$. As expected based on our use of Dmel as the "gold standard", there was no significant improvement in reads mapping exclusively to the annotation in Dmel. This 
A

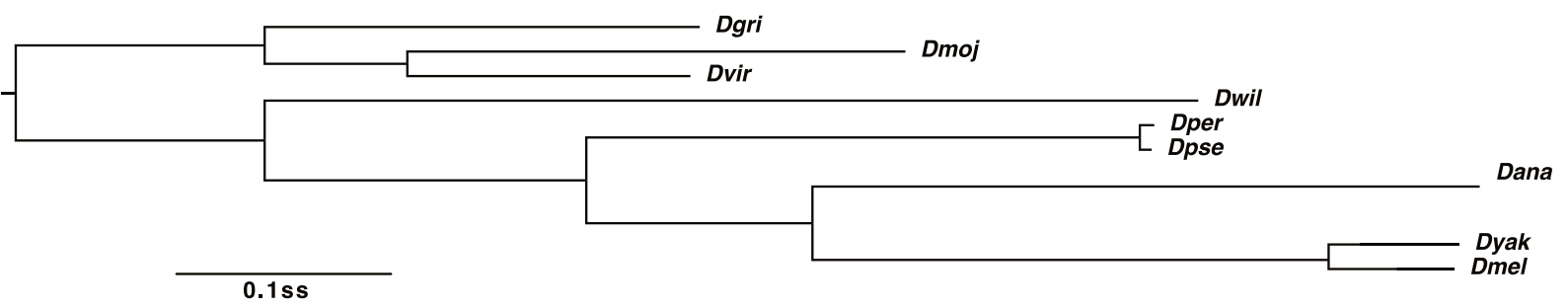

B

$400 \mathrm{bp}$

$1000 \mathrm{bp}$

$200 \mathrm{bp}$

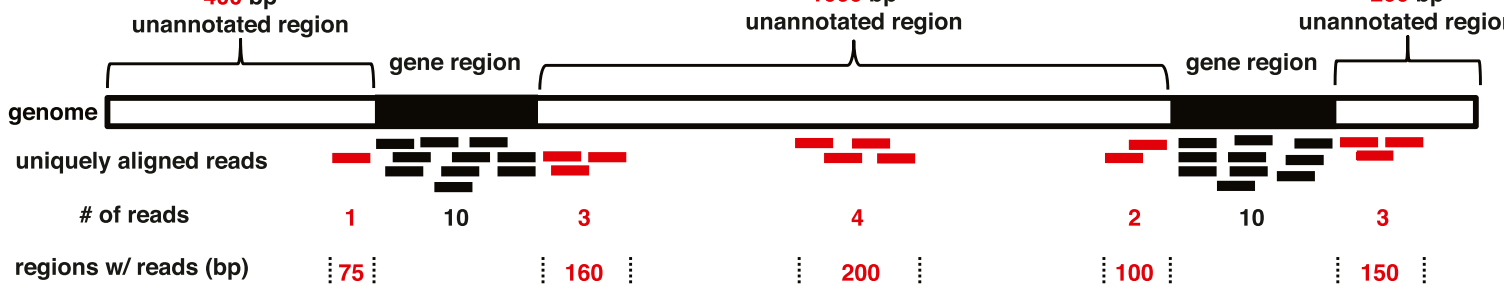

$\%$ Uniquely mapped

reads in unannotated $=\frac{1+3+4+2+3}{1+10+3+4+2+10+3}=39 \%$ regions

\% Uniquely mapped reads in unannotated regions
Female Male Dmel Dyak Dana Dpse Dper Dwil Dmoj Dvir Dg

C

D \% Unannotated regions with uniquely mapped reads

\begin{tabular}{c|c|c|c|c|c|c|c|c|c|} 
Organism & 1 & 4 & 5 & 5 & 28 & 9 & 5 & 5 & 28 \\
\cline { 2 - 11 } Organism & 1 & 4 & 6 & 6 & 31 & 11 & 8 & 7 & 31 \\
\hline
\end{tabular}

\begin{tabular}{c|c|c|c|c|c|c|c|c|c|} 
Gonad & 1 & 3 & 4 & 5 & 26 & 8 & 5 & 5 & 29 \\
\cline { 2 - 10 } & 1 & 5 & 6 & 6 & 28 & 16 & 8 & 7 & 34 \\
\hline
\end{tabular}

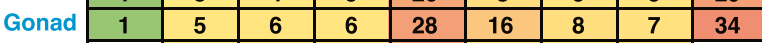

\begin{tabular}{c|c|c|c|c|c|c|c|c|c|} 
Repr. Tract & - & 4 & 5 & 5 & 27 & 8 & 4 & 5 & - \\
\cline { 2 - 15 } & - & 9 & 24 & 14 & 46 & 15 & 11 & 11 & - \\
\hline
\end{tabular}

\begin{tabular}{c|c|c|c|c|c|c|c|c|c|}
\multirow{2}{*}{ Repr. Tract } & - & 9 & 24 & 14 & 46 & 15 & 11 & 11 & - \\
\cline { 2 - 10 } & 1 & - & - & - & - & - & - & - & 26 \\
\cline { 2 - 7 } & & -
\end{tabular}

\begin{tabular}{c|c|c|c|c|c|c|c|c|c|}
\hline Int. Repr. Tract & 1 & - & - & - & - & - & - & - & 26 \\
\hline
\end{tabular}

Int. Repr. Tract 2

Terminalia 1

Terminalia

Thorax 1

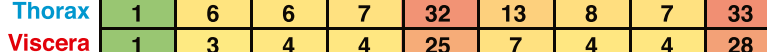

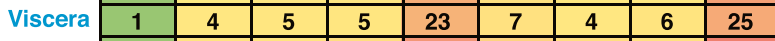

\begin{tabular}{l|l|l|l|l|l|l|l|l|l|} 
Head & 1 & 6 & 6 & 8 & 31 & 12 & 8 & 7 & 37 \\
\cline { 2 - 11 } & 1 & 6 & 6 & 9 & 34 & 11 & 9 & 9 & 37
\end{tabular}

\begin{tabular}{|c|c|c|c|c|c|c|c|c|c|} 
Head & 1 & 6 & 6 & 9 & 34 & 11 & 9 & 9 & 37 \\
\cline { 2 - 10 } & 1 & 3 & 4 & 5 & 24 & 9 & 4 & 5 & 30 \\
\hline
\end{tabular}

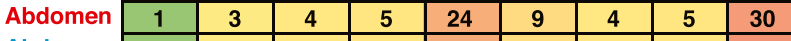

Abdomen

Dmel Dyak Dana Dpse Dper Dwil Dmoj Dvir Dgri

\begin{tabular}{|c|c|c|c|c|c|c|c|c|}
\hline 6 & 12 & 12 & 14 & 33 & 23 & 16 & 14 & 35 \\
\hline 8 & 13 & 15 & 16 & 35 & 25 & 20 & 17 & 35 \\
\hline 6 & 11 & 12 & 15 & 32 & 22 & 13 & 17 & 40 \\
\hline 14 & 20 & 20 & 17 & 31 & 32 & 20 & 17 & 38 \\
\hline- & 9 & 11 & 12 & 33 & 21 & 13 & 14 & - \\
\hline- & 13 & 14 & 18 & 32 & 24 & 12 & 17 & - \\
\hline 7 & - & - & - & - & - & - & - & 31 \\
\hline 8 & - & - & - & - & - & - & - & 35 \\
\hline 6 & - & - & - & - & - & - & - & 36 \\
\hline 5 & - & - & - & - & - & - & - & 33 \\
\hline 8 & 12 & 13 & 16 & 34 & 25 & 14 & 13 & 33 \\
\hline 10 & 16 & 18 & 15 & 36 & 27 & 16 & 14 & 33 \\
\hline 6 & 10 & 12 & 10 & 32 & 20 & 11 & 13 & 35 \\
\hline 9 & 14 & 16 & 17 & 31 & 26 & 12 & 16 & 32 \\
\hline 11 & 14 & 14 & 18 & 38 & 29 & 18 & 18 & 37 \\
\hline 13 & 15 & 17 & 20 & 39 & 29 & 19 & 21 & 37 \\
\hline 7 & 10 & 11 & 18 & 33 & 22 & 13 & 18 & 33 \\
\hline 6 & 10 & 12 & 18 & 31 & 21 & 13 & 16 & 32 \\
\hline
\end{tabular}

E

\begin{tabular}{|c|c|c|c|c|c|c|c|c|c|}
\hline Organism & 1 & 1 & 1 & 1 & 2 & 1 & 1 & 1 & 1 \\
\hline Organism & 1 & 1 & 1 & 1 & 3 & 1 & 1 & 1 & 1 \\
\hline Gonad & 1 & 1 & 1 & 1 & 2 & 1 & 1 & 1 & 1 \\
\hline Gonad & 1 & 2 & 2 & 1 & 2 & 2 & 1 & 1 & 2 \\
\hline Repr. Tract & - & 1 & 1 & 1 & 3 & 1 & 1 & 1 & - \\
\hline Repr. Tract & - & 1 & 1 & 2 & 3 & 1 & 1 & 2 & - \\
\hline Repr. Tract & 1 & - & - & - & - & - & - & - & 1 \\
\hline Repr. Tract & 1 & - & - & - & - & - & - & - & 1 \\
\hline Terminalia & 1 & - & - & - & - & - & - & - & 1 \\
\hline Terminalia & 1 & - & - & - & - & - & - & - & 1 \\
\hline Thorax & 1 & 1 & 1 & 1 & 3 & 2 & 1 & 1 & 1 \\
\hline Thorax & 1 & 2 & 2 & 2 & 3 & 2 & 2 & 1 & 1 \\
\hline Viscera & 1 & 1 & 1 & 1 & 2 & 1 & 1 & 1 & 1 \\
\hline Viscera & 1 & 1 & 1 & 2 & 2 & 1 & 1 & 1 & 0 \\
\hline Head & 1 & 1 & 1 & 2 & 4 & 2 & 2 & 2 & 2 \\
\hline Head & 1 & 1 & 2 & 3 & 4 & 2 & 2 & 3 & 2 \\
\hline Abdomen & 1 & 0 & 1 & 2 & 3 & 1 & 1 & 2 & 1 \\
\hline Abdomen & 1 & 1 & 1 & 2 & 4 & 1 & 1 & 1 & 1 \\
\hline
\end{tabular}

$\mathbf{F}$

\begin{tabular}{|c|c|c|c|c|c|c|c|c|}
\hline 5 & 7 & 6 & 8 & 10 & 8 & 9 & 9 & 11 \\
\hline 8 & 8 & 9 & 10 & 12 & 10 & 13 & 11 & 11 \\
\hline 5 & 7 & 6 & 10 & 9 & 8 & 7 & 11 & 16 \\
\hline 13 & 15 & 14 & 11 & 11 & 18 & 14 & 11 & 18 \\
\hline- & 5 & 5 & 7 & 10 & 7 & 7 & 9 & - \\
\hline- & 9 & 8 & 12 & 9 & 10 & 6 & 12 & - \\
\hline 6 & - & - & - & - & - & - & - & 9 \\
\hline 7 & - & - & - & - & - & - & - & 12 \\
\hline 6 & - & - & - & - & - & - & - & 12 \\
\hline 5 & - & - & - & - & - & - & - & 10 \\
\hline 8 & 7 & 8 & 11 & 9 & 11 & 7 & 8 & 8 \\
\hline 10 & 11 & 13 & 9 & 13 & 14 & 10 & 9 & 9 \\
\hline 5 & 5 & 6 & 5 & 9 & 6 & 6 & 8 & 11 \\
\hline 9 & 9 & 11 & 11 & 9 & 12 & 6 & 11 & 8 \\
\hline 10 & 9 & 8 & 12 & 14 & 13 & 11 & 13 & 13 \\
\hline 13 & 11 & 11 & 14 & 16 & 14 & 12 & 16 & 13 \\
\hline 7 & 5 & 5 & 13 & 11 & 8 & 7 & 13 & 9 \\
\hline 6 & 6 & 7 & 12 & 9 & 9 & 7 & 11 & 8 \\
\hline
\end{tabular}


harmonized annotation will provide an improved basis for comparative genomics studies. These annotations provide transcriptlevel features on par with those of Dmel (Table 1). We also provided annotation of all species as gtf/gff files in the GSE99574 and GSE80124 supplements so that researchers can make immediate use of this update.

\section{Summary of new gene models}

Because the original annotation for the non-melanogaster members focused on conserved longest ORFs at each locus (Drosophila 12 Genomes Consortium et al, 2007), we anticipated that much of the improvement to the annotation would come from extending the annotation of UTRs and new isoforms because of alternative promoters, termination, and alternative splicing, as well as noncoding or minimally coding RNAs (ncRNAs). Indeed, we found that $\sim 8,000$ new gene models per species overlapped with and extended the older annotations (Fig 2A). For example, the Dwil gene GK27243, which is expressed in the testis, had the same splice junctions in the old and new annotation (YOgnWI09161), but had longer 5'- and $3^{\prime}$-ends in the updated annotation (Fig 2B). We also observed an increase of 10,000-20,000 isoforms in the new annotation compared with the old one (Fig 2C). For example, the Dper doublesex (dsx) locus (GL23549) had a single annotated isoform (Fig 2D). This is unlikely to be correct, as the dsx function is highly conserved ( $Y i$ \& Zarkower, 1999) and the $d s x$ locus encodes sex-specific transcription factors from sex-specifically spliced pre-mRNAs (Burtis \& Baker, 1989). Our new annotation (YOgnPE00925) captured sex-specific isoforms of Dper dsx and includes a new upstream promoter. We also observed 700-1,300 instances per species where gene models were merged (Fig 2E). In at least some cases, this was strongly supported by expression data. For example in the case of the YOgnWI03804 locus, the last two exons of Dwil GK26840 are clearly joined by junction reads to the single exon of GK20038 locus forming an updated gene model (Fig 2F). However, we did observe 700 instances of merging in the well-annotated Dmel genome, which seems excessive. Overall, we generated 1,000-2,000 completely novel annotations per species (Fig $2 \mathrm{G}$ and $\mathrm{H}$ ). These included ncRNAs ( $24 \%$ of novel annotations), such as the Dyak noncoding homolog (YOgnYA12879) of rna on X 1 (roX1). Dmel roX1 is a component of the male-specific $X$-chromosome dosage compensation complex (Kuroda et al, 2016), and like the Dmel ortholog, the Dyak roX1 locus is expressed in males, but not females. Loci-producing ncRNAs tend to diverge rapidly, but both the Dmel and Dyak roX1 loci are flanked by the yin and echinus orthologs. The combination of sequence, expression pattern, and synteny strengthen the conclusion that these roX1 genes descended from a common ancestral gene.

To identify other novel orthologs such as roX1, we analyzed the synteny, sex- and tissue-biased expression patterns, and gene
Table 1. Number of features in the new annotations.

\begin{tabular}{llll} 
& Genes & Transcripts & Exons \\
\hline Dmel $^{\mathrm{a}}$ & 17,730 & 35,105 & 83,251 \\
\hline Dyak & 16,473 & 36,082 & 96,353 \\
\hline Dana & 16,029 & 32,808 & 91,197 \\
\hline Dpse & 16,441 & 39,527 & 103,772 \\
\hline Dper & 17,726 & 35,392 & 97,224 \\
\hline Dwil & 15,843 & 30,308 & 89,256 \\
\hline Dmoj & 14,699 & 33,272 & 94,364 \\
\hline Dvir & 15,074 & 33,357 & 93,631 \\
\hline Dgri & 16,605 & 32,313 & 93,800 \\
\hline
\end{tabular}

abased on FlyBase annotation (release 2017_03).

structures of previously identified orthologs of Dmel genes and developed a SVM to generate a list of candidate orthologs, which were then compared at the sequence similarity level (see the Materials and Methods section). We conservatively called 500-1,000 new 1:1 orthologs per species (Fig 3A). They have on average two transcripts per gene and around three introns per transcript (Table 2). Their ratios of alignable regions with $\mathrm{Dmel}$ transcripts ranges from 0.69 to 0.81 and the ratio of identical bases ranges from 0.55 to 0.88 , depending on the particular non-melanogaster species (Table 2). For example, we found orthologs of the noncoding Dmel CR42860 gene in four of the eight other species (YOgnYA06038, YOgnAN10714, YOgnWI07915, and YOgnVI13637 in Dyak, Dana, Dwil, and Dvir, respectively; Fig 3B). In each case, the CR42860 ortholog is most strongly expressed in the thorax. Interestingly, in Dwil, YOgnWI07915 also showed female-biased expression $\left(P_{\text {adj }}=5.4 \times 10^{-10}\right.$, DESeq2), highlighting the fact that we can observe changes in sexand/or tissue-biased expression in the phylogeny. Extending the Drosophila orthology to include ncRNAs should allow for the exploration of conserved and divergent functions of this understudied aspect of comparative genomics. In the future, a more formalized and generalized pipeline could be developed to extend this ortholog-finding methodology as a general tool.

\section{CDNA validation}

Because we used the illumina RNA-seq data to build the new annotations, we needed an independent transcriptome dataset for validation. The Dgri annotation was greatly changed in our work and has been unexplored at the RNA-seq level. We therefore chose to validate a subset of the Dgri annotation. Our logic was that if we can validate the methodology using the most radically updated annotation, then the prospects for the rest of annotations are

\section{Figure 1. Evaluation of re-annotation for non-melanogaster Drosophila species.}

(A) Bayesian phylogenetic tree of nine Drosophila species. Nodes are supported by $100 \%$ posterior probabilities, and phylogenetic distance is shown as substitutions per site (ss). After (Chen et al, 2014). Standard three letter abbreviations are used (see text). (B) Cartoon of measurements of reads mapped to unannotated regions. Gene regions (fill), unannotated regions (open), RNA-seq reads (bars) mapped to gene regions (black) or unannotated regions (red) are shown. Numbers of reads (\#), regions (in bps, flanked by dotted lines) with reads, and examples of calculating the ratios of unannotated reads are also shown. Percentage of reads (C, E) and regions with mapped reads (D, F) in unannotated regions before (C, D) and after (E, F) re-annotation. Green, yellow, and red denote low, median, and high percentage, which are also indicated in each cell. Expression profile tissues are shown (left) for female (red) and male (blue) samples. The reproductive (Repr.) tract includes the internal ducts and glands and the terminalia. The internal reproductive (Int. Repr.) tract does not include the terminalia. Missing tissues are shown (dash and open fill). 
A

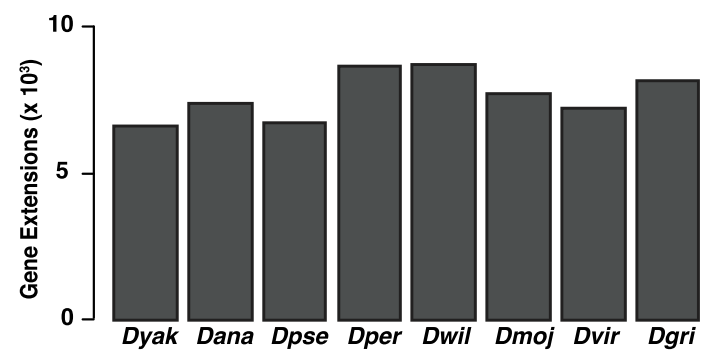

B

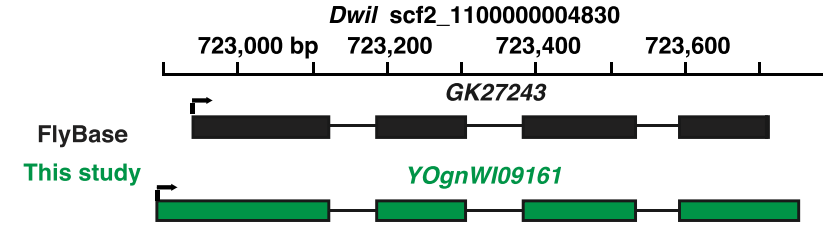

Female Male

Organism

Organism

Gonad

Gonad

Repr. Tract

Repr. Tract

Thorax

Thorax

Viscera

Viscera

Head

Head

Abdomen

Abdomen

E

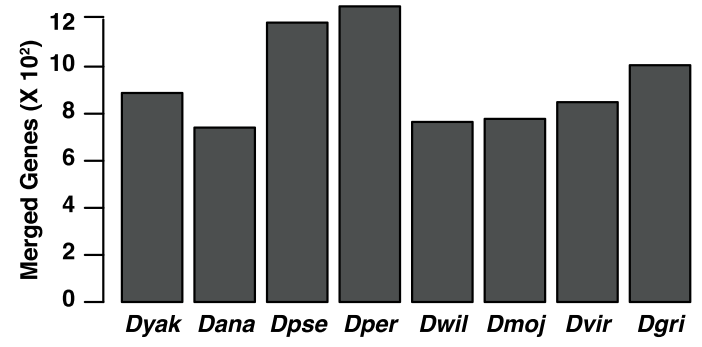

F

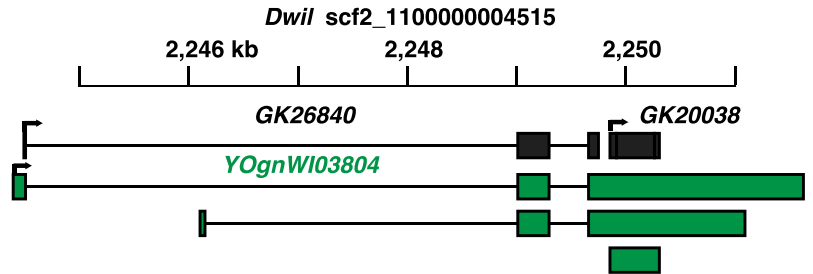

Female Male

Organism

Organism

Gonad

Gonad

Repr. Tract

Repr. Tract

Thorax

Thorax

Viscera

Viscera

Head

Head

Abdomen

Abdomen

C

D

G

an and a

(an) Mnsm

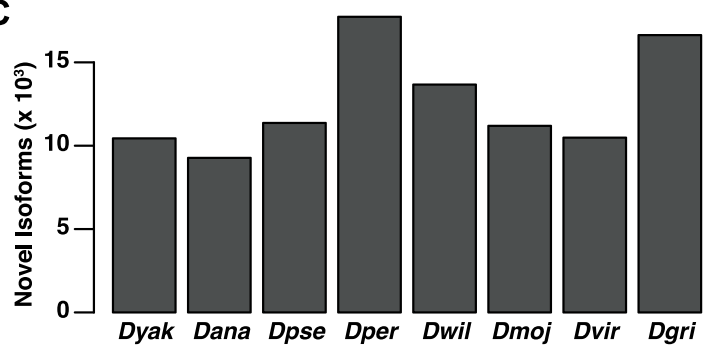

Dper scaffold_o
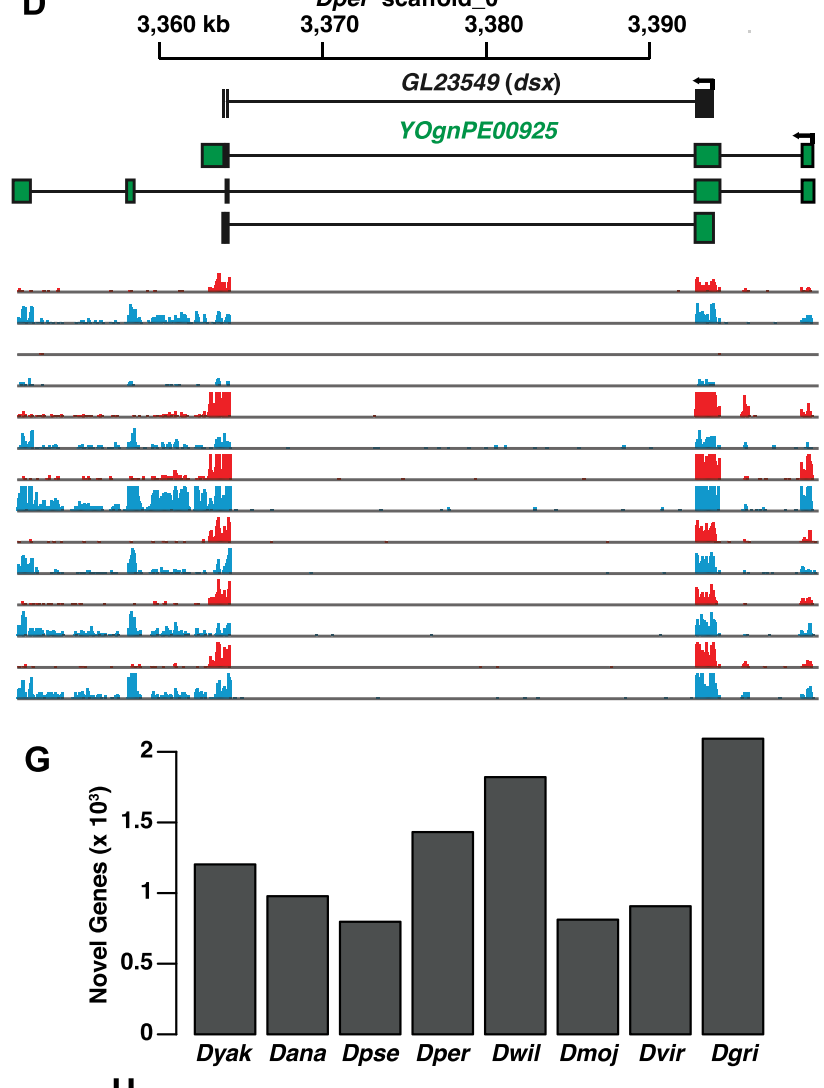

H

Dyak chr X

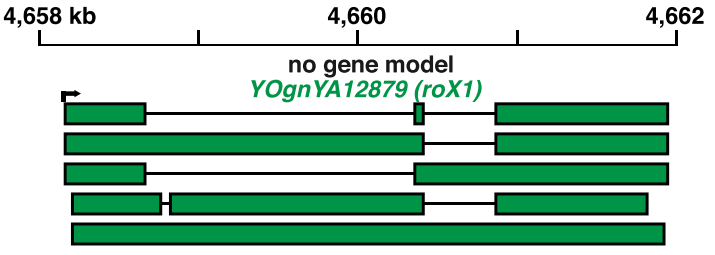

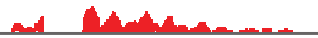

an

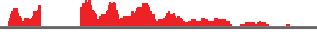

an and -

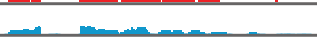

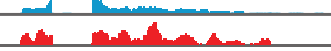

Aw man

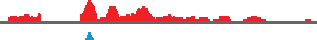

and

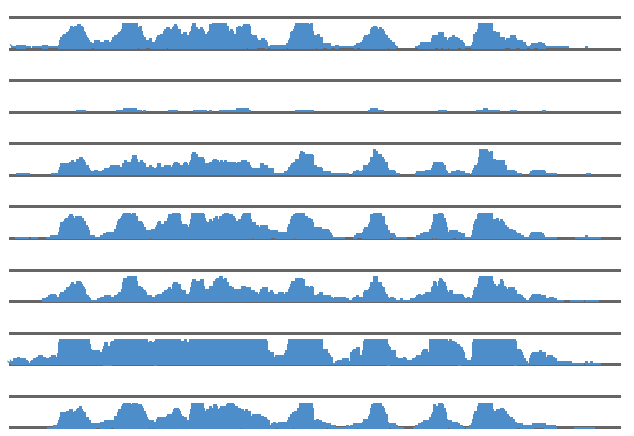


A

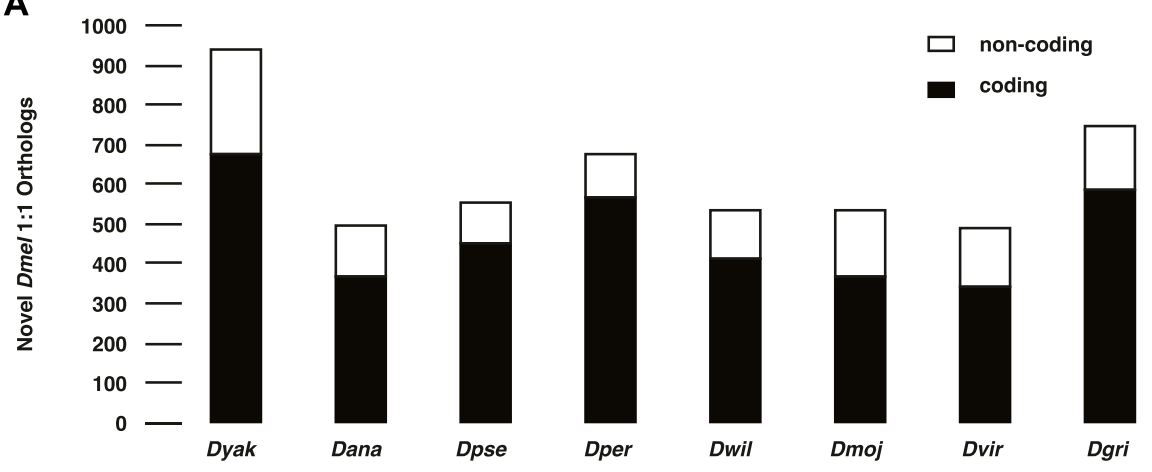

Figure 3. Summary and novel 1:1 orthologs

(A) Number of novel Dmel 1:1 orthologs in each of the eight non-melanogaster species. The coding (filled) and noncoding (open) status of genes in Dmel are.

(B) An example of novel 1:1 orthologs of Dmel CR42860 identified in Dyak, Dana, Dwil, and Dvir. CR42860 is nested in the intron of sls in all five species. Significant $\left(P_{a d j}=5.4 \times 10^{-10}\right)$ female-biased expression of the Dwil ortholog is shown (asterisk). See Fig 2 for abbreviations and color coding.

\section{B}

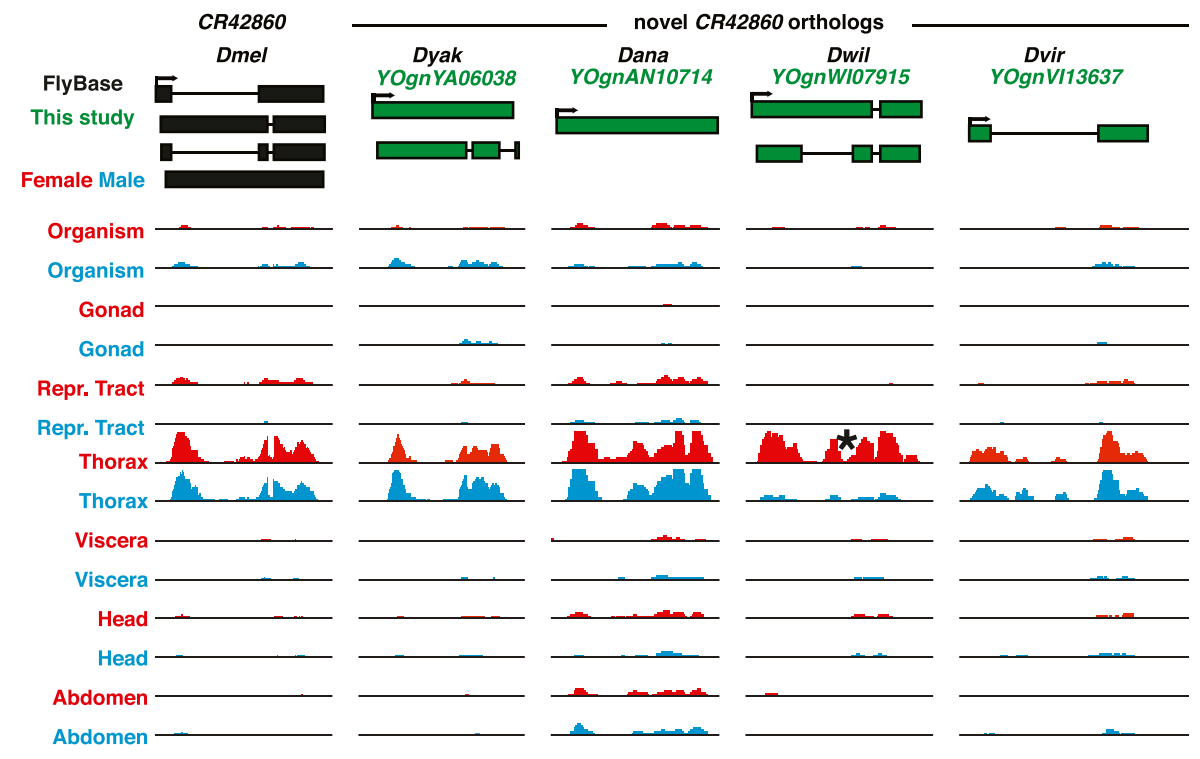

outstanding. Longer reads have a distinct advantage for this validation, as they can capture the alternative splice forms without reliance on short junction reads. Therefore, we conducted duplicate PolyA+ PacBio Iso-seq for Dgri sexed adults. To better sample the transcriptome, we fractionated each RNA preparation into three size categories (see the Materials and Methods section). We generated 288,575 high-quality, full-length, non-chimeric, cDNAs and compared overlap with the annotation. This sampling experiment covered $27-29 \%$ of the FlyBase and new annotations (Fig 4A).

To systematically analyze the relationship between the cDNA sequences and the annotations, we measured the intersection/ union (Jaccard index) for base coverage at the transcript isoform level (Fig 4B). The most dramatic difference in the Jaccard index was at zero, where there is no overlap between cDNAs and annotations.
We observed a dramatic decrease in the fully nonoverlapping cDNAs when we used the new annotation. The distribution of the Jaccard index scores shows a shift towards full overlapping, with $56 \%$ of cDNA/new annotations showing $75-100 \%$ similarity. For example, four cDNAs mapping to the Dgri GH16482 locus (Jaccard index $>0.94$ ) validated the three isoforms of YOgnGR06478, with three distinct promoters, each of which has an extended 3'-UTR relative to the FlyBase annotation (Fig $4 \mathrm{C}$ ). Similarly, we found support (Jaccard index >0.95) for the previously unannotated locus YOgnGR03214 (Fig 4D).

In addition to providing transcriptome data from another independent platform, the PacBio cDNAs also provided us with an opportunity to evaluate the potential improvements that can be made to our updated annotation. Although an impressive $78 \%$ of

Figure 2. Summary and re-annotation.

(A) Number of new gene annotations with extended coverage and (B) one example. (C) Number of novel isoforms in the re-annotation and (D) one example. (E) Number of merged genes in the re-annotation and (F) one example. (G) Number of novel genes in the re-annotation, and (H) one example. (A-H) Species, Scaffold IDs, and locus names are shown. FlyBase gene models (black) and new models (green) are shown. Orientation of transcripts are shown (arrow at 5'-end). Expression level tracks (arbitrary FPKM scale) for indicated tissues/sexes are shown. 
Table 2. Features of novel 1:1 orthologs to Dmel.

\begin{tabular}{lllll} 
& Transcripts per gene & Introns per transcript & Alignable/total & Identical/alignable \\
\hline Dmel $^{\text {a }}$ & 2.0 & 4.3 & - & 0.8 \\
\hline Dyak & 2.0 & 3.3 & 0.7 & 0.9 \\
\hline Dana & 1.8 & 2.2 & 0.7 & 0.6 \\
\hline Dpse & 2.4 & 3.8 & 0.7 & 0.6 \\
\hline Dper & 2.3 & 2.6 & 0.7 & 0.6 \\
\hline Dwil & 1.8 & 2.3 & 0.7 & 0.6 \\
\hline Dmoj & 2.2 & 2.7 & 0.7 & 0.6 \\
\hline Dvir & 1.8 & 2.2 & 0.7 & 0.5 \\
\hline Dgri & 1.9 & 2.6 & & 0.6 \\
\hline
\end{tabular}

a Based on FlyBase annotation (release 2017_03).

288,575 cDNA are fully contained in our annotation, $24 \%$ had transcript ends beyond our annotation (5\% with $5^{\prime}$ end extension, $4 \%$ with $3^{\prime}$ end extension, $14 \%$ with extension at both $5^{\prime}$ and $3^{\prime}$ ends, and $<1 \%$ within intergenic regions) (Fig $4 \mathrm{E}$ ). When looking at the 236,779 intron-containing cDNAs, we found that $87 \%$ had junctions compatible with our annotation (78\% with all junctions present and $10 \%$ with called junctions annotated) and only $13 \%$ with novel junctions (1\% with all junctions unannotated, $12 \%$ with junctions partially unannotated, $<1 \%$ with antisense junctions, which are probably reverse transcription errors) (Fig 4F). Overall, these cDNAs suggest that the improvements in the annotations are substantial, but also highlight the ongoing need for annotation updates.

\section{Discussion}

Previous work has used expression data in the Drosophila genus to validate the gene models in Dmel (Drosophila 12 Genomes Consortium et al, 2007; Chen et al, 2014), but there has been less systematic effort to use the knowledge from Dmel to inform and annotate the rest of the genus. To maximize the value of the sequenced Drosophila genomes, we have generated an extensive expression profile to assemble transcript models and update the annotations. In all cases, this resulted in an extensive set of new predicted transcripts. To leverage the decades of dedicated annotation that has been performed on Dmel (Adams et al, 2000; Celniker, 2000; Lewis et al, 2002; Misra et al, 2002; Celniker \& Rubin, 2003; Drysdale, 2003; Ashburner \& Bergman, 2005; Brown \& Celniker, 2015), we generated thousands of de novo Dmel annotations, to determine optimal parameters and filters that resulted in the best match to the existing Dmel annotation. We then applied this optimized set of parameters to the rest of the genomes. This was largely successful, as we not only generated tens of thousands of new isoform models in each species, but we were also able to validate these models in Dgri with an independent set of full-length cDNAs. We suggest that when there is a high-quality annotation of a given species, this methodology could be used to tune the annotation pipelines for related species. The fact that this worked well for a species, Dgri, that is separated from Dmel by about 40 million years (Leung et al, 2015), suggests that targeting a few genomes in a lineage for full curation, can then result in a high-quality annotation for scores of related species.

The products of this article are the annotations. We hope that they are widely used by the evolutionary and comparative genomics communities. Given how often Drosophila are used in evolutionary studies on gene birth, death, duplication, and divergence, having improved annotations will facilitate a large number of studies. Similarly, workers interested in understanding Dmel genes should find these annotations useful for determining if a given feature is evolutionarily conserved. For example, this should be particularly true for identifying ncRNAs and RNAi-binding protein-binding sites in UTRS (Cech \& Steitz, 2014), as those features were lacking for most non-Dmel genes. To make these annotations as useful as possible, we have posted the gene models and data in a number of formats (such as gtf, gff, bigWig, gene-level normalized read counts by DESeq2, and transcript-level Transcripts Per Million by Salmon). Some of these species will undergo reassembly in the future. The annotations for new assemblies will be relatively easy to update, without new experiments, as we saved all the unmapped reads (GSE99574 and GSE80124) at the sequence read archive (Leinonen et al, 2011).

There are efforts to sequence much of the tree of life, but manual annotation of those genomes would be prohibitively expensive. A more focused annotation effort on a key species in a phylogeny could be leveraged to annotate an entire phylogeny. We will need additional examples from other phylogenies to determine how sparsely high quality annotation will be needed, but within the species we examined here, our data suggests that organisms separated by tens of millions of years can have improved annotations using our method. The collective efforts from multiple groups in a genomics community could create reference annotations of large sets of genomes with a combination of focused effort on a single-type species and propagation of the annotation to the rest of the phylogeny.

\section{Materials and Methods}

See the extensive reagent and resources table for strains, media, reagents and suppliers, software, database submissions, and other identifiers (Table S1). 
A

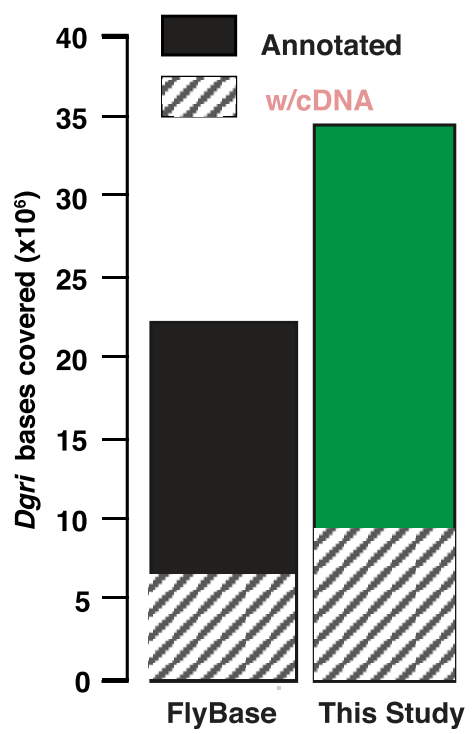

B

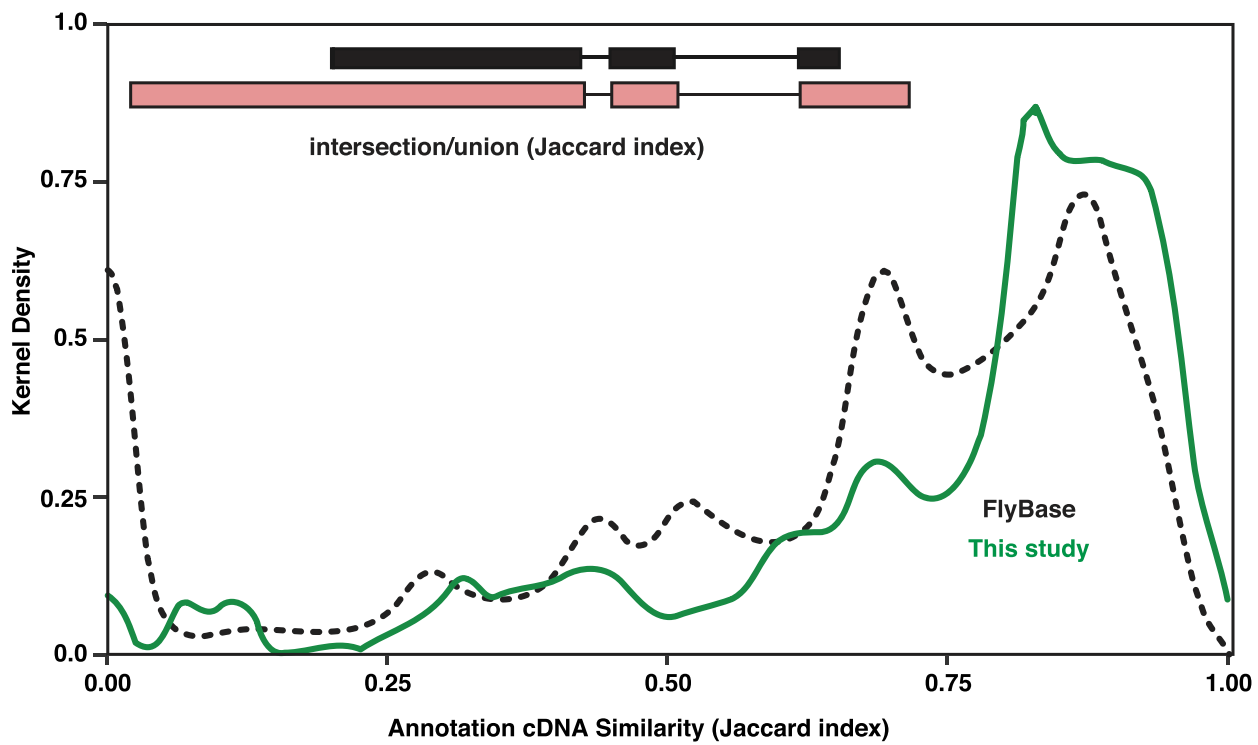

C

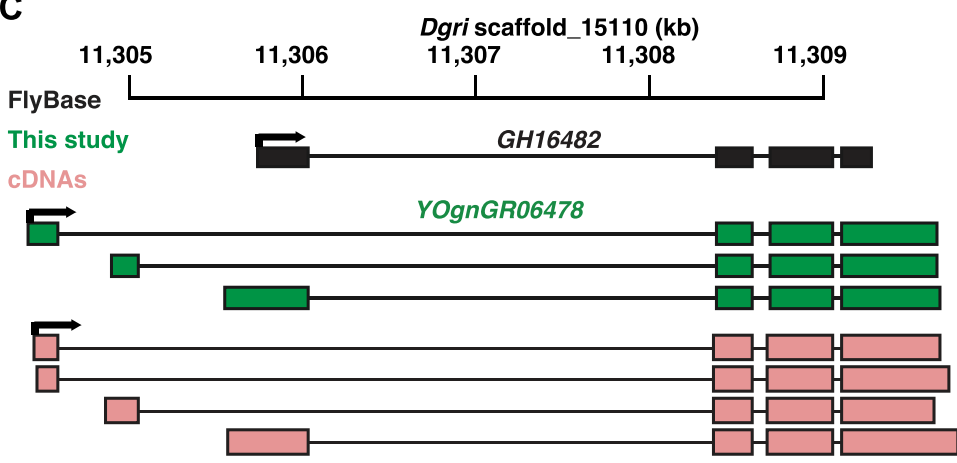

D

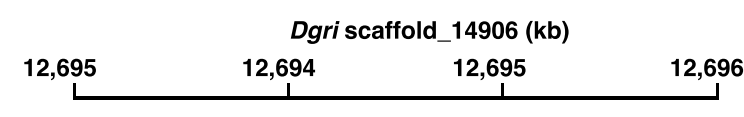

no gene model
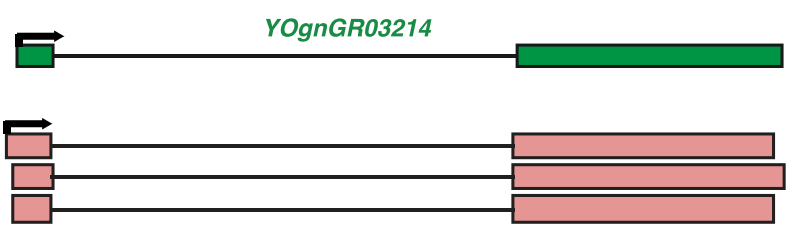

E
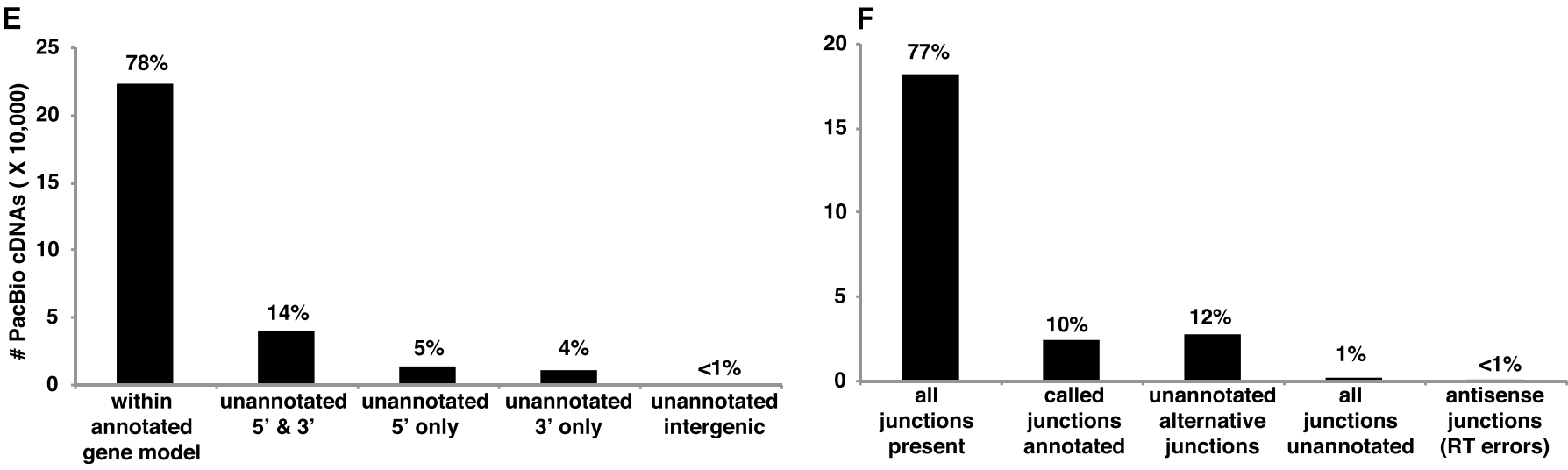

Figure 4. PacBio cDNA validation of $D$. grimshawi annotation.

(A) Bases in the genome covered by annotations (whole bars) and validated by PacBio Iso-seq cDNA sequencing (stripes) in Dgri. (B) Similarity between annotated gene models and cDNAs before (dotted black line) and after re-annotation (solid green line) as measured by the Jaccard index (intersection/union), as illustrated (inset). The Jaccard index distributions are shown in kernel density. (C) An example of cDNA (SRR6840922) aligning to a previous and new gene model, where the new gene models have extended coverage and a greater Jaccard index. (D) An example of cDNAs (SRR6840922) aligning with a novel gene model and a Jaccard score of "0" with the previous annotation. See Fig 2 for abbreviations and color coding. (E) Number and percentage of cDNAs within the annotated gene model, cDNAs with unannotated 5' and $3^{\prime}$ ends, cDNAs with unannotated 5' end only, cDNAs with unannotated $3^{\prime}$ end only, and cDNAs within the intergenic region. (F) Number and percentage of cDNAs with all junctions present in annotation (only intron-containing CDNA used in this analysis), cDNAs with called junctions annotated, cDNAs with unannotated alternative junctions, cDNAs with all junctions unannotated, and cDNAs with antisense junctions (probably because of reverse transcription error). 


\section{Flies}

Flies were grown at the National Institutes of Health (Dmel), the Drosophila Species Stock Center at University of California San Diego (Dyak, Dana, Dpse, Dper, Dwil, Dmoj, and Dvir), or the Hawaiian Drosophila Research Stock Center (Dgri). Growth conditions are given in GEO GSE99574 and GSE80124. For each sex and species, we dissected seven to eight adult tissues or body parts in PBS and transferred the samples immediately to RNAlater.

\section{RNA-seq}

Illumina sequencing details are given in GEO (GSE99574 and GSE80124) and their GSM (i.e., sample accessions number) subpages. Briefly, we isolated RNA using the RNeasy 96 kit. We added External RNA Controls Consortium (ERCC) spike-ins (Jiang et al, 2011; Zook et al, 2012) for quality control purposes. We conducted singleend stranded 76-bp polyA ${ }^{+}$RNA-seq experiments for all samples using the TruSeq kit and protocol. We quantified nucleic acids with Quant-iT RiboGreen or PicoGreen kits. We multiplexed using both index adaptors and mixing RNA from distantly related species and sequenced on the HiSeq2000 Sequencing System. The use of species multiplexing has been used previously (Paris et al, 2015). De-multiplexed reads were produced by Illumina CASAVA (v1.8.2) as fastq files. We mapped the reads of mixed species libraries with HiSAT2 (v2.0.5; - dta and -max-intronlen $=300,000$ ) and used SAMtools (v0.1.19) (Li et al, 2009) to sort the HiSAT2-generated bam files by read name. We used a python script demultiplexer (v1.0) to scan the bam file to collect the reads specific to one species or ERCC spike-ins (Jiang et al, 2011; Zook et al, 2012), and ambiguous reads, also provided in GEO. We converted from bam to fastq format using BEDTools (v2.25.0; bamtofastq) (Quinlan \& Hall, 2010). We used the most current annotations of the species (FlyBase release 2017_03), except for Dgri because NCBI and FlyBase used our prepublication RNA-seq data to improve the annotation of Dgri using gnomon (Kapustin et al, 2008). We used the last version of Dgri annotation, before RNA-seq data inclusion (Flybase release 2016_05). The median Spearman's $r$ of log-transformed gene expression among different biological replicates is above 0.95 .

PacBio CDNA details are found at NCBI Sequence Read Archive (SRA) (SRP135764). Briefly, we constructed Dgri cDNA libraries following the isoform sequencing (Iso-Seq) protocol using the Clontech SMARTer cDNA Synthesis Kit and the SageELF sizeselection system. We used $500 \mathrm{ng}$ total RNA per reaction for the polyA+ enrichment and first strand synthesis, and conducted the first round of PCR amplification $\left(95^{\circ} \mathrm{C}\right.$ for $2 \mathrm{~min}$; 14 cycles of $98^{\circ} \mathrm{C}$ for $20 \mathrm{~s}, 65^{\circ} \mathrm{C}$ for $15 \mathrm{~s}, 72^{\circ} \mathrm{C}$ for $4 \mathrm{~min} ; 72^{\circ} \mathrm{C}$ for $5 \mathrm{~min}$ ) to generate doublestrand $\mathrm{CDNA}$ for size selection. We used three fraction ranges (SageELF index 10-12 or 1-2 kb, 8-9 or 2-3 kb, and 5-7 or 3-5 kb) of double-strand CDNA for the second round of PCR amplification $\left(95^{\circ} \mathrm{C}\right.$ for $2 \mathrm{~min}$; $\mathrm{N}$ cycles of $98^{\circ} \mathrm{C}$ for $20 \mathrm{~s}, 65^{\circ} \mathrm{C}$ for $15 \mathrm{~s}, 72^{\circ} \mathrm{C}$ for $\mathrm{X}$ min; $72^{\circ} \mathrm{C}$ for $5 \mathrm{~min}$ ). We repaired DNA ends and performed blunt-end ligation. We quantified SMRTbell libraries by Qubit Fluorometric Quantitation and qualified by Bioanalyzer beforesequencing on the PacBio RS II using DNA Sequencing Reagent kit 4.0 v2 with a run time of $240 \mathrm{~min}$. We used sixth generation polymerase and fourth generation chemistry (P6-C4). Circular consensus (ccs2) reads
(-maxLength $=40,000$-minPasses $=1$ ) were generated using PacBio pitchfork (v0.0.2) after conversion of bax.h5 files to bam using bax2bam. The final full-length nonchimeric Iso-seq reads were concatenated from three fractions and available in SRA (SRP135764).

\section{Annotation optimization}

We developed a method to match the existing Dmel annotation with de novo RNA-seq data (Fig 5A). We mapped reads with HiSAT2 (v2.0.5; -dta and -max-intronlen $=300,000$ ) (Kim et al, 2015). We then used StringTie (v1.3.3) (Pertea et al, 2015, 2016) to generate de novo annotation using the bam alignments from HiSAT2. We set minimum transcript length according to the shortest gene in Dmel (i.e., $30 \mathrm{bp}$ ), and we set the strandedness library to "--rf". We optimized "-c" (minimum reads per bp coverage to consider for transcript assembly), "-g" (minimum gap between read mappings triggering a new bundle), "f" (minimum isoform fraction), "-j" (minimum junction coverage), "-a" (minimum anchor length for junctions), and "-M" (maximum fraction of bundle allowed to be covered by multihit reads). We used a union set of reads from replicated $w^{1118}$ and OreR females and males to optimize StringTie parameters. To test which combination of parameters generated the de novo annotation closest to FlyBase, we used the Jaccard index (BEDTools v2.25.0) of unique exons to measure similarity. The use of Jaccard index is meant to eventually maximize the intersection between simulated annotation and FlyBase one and meanwhile minimize the union. In the first round of testing (Fig 5B), we tested all combinations of " $c$ " $(1,3,5,7,9)$, " $g$ " $(10,30,50,70,90)$, "f" $(0.01,0.03$, $0.05,0.07,0.09)$, “j” (1, 2, 3, 5, 7, 9), “a” (5, 10, 15, 20, 25), and “M" $(0.1,0.3$, $0.5,0.7,0.9)$. Among the 15,625 tests, the parameters with the highest Jaccard index were " $c "=3, " g$ " $=50, " f "=0.01, " j "=3, " a "=10$, and " $M "=$ 0.9. In the second round of testing, we further picked all combinations of the points next to the previous optimal parameters with smaller intervals-“ $c$ " $(1,1.5,2,2.5,3,3.5,4,4.5,5), " g$ " $(30,40,50,60$, 70), “f” (0.005, 0.01, 0.015, 0.02, 0.025, 0.03), “j” (1, 2, 3, 4, 5), “a” (5, 6, 7, 8, $9,10,11,12,13,14,15)$, and " $M$ " (0.7, 0.75, 0.8, 0.85, 0.9, 0.95). Among the 89,100 tests, the parameters with the highest Jaccard index were $" c "=1.5, " g "=50, " f "=0.015, " j "=1, " a "=14$, and " $M "=0.95$. In the third round of test, we further picked all combinations of the points next to the previous optimal parameters with smaller intervals- " $c$ " (1, $1.1,1.2,1.3,1.4,1.5,1.6,1.7,1.8,1.9,2)$, "g" $(40,41,42,43,44,45,46,47,48$, $49,50,51,52,53,54,55,56,57,58,59,60)$, “f” (0.01, 0.011, 0.012, 0.013, $0.014,0.015,0.016,0.017,0.018,0.019,0.02)$, "j" $(1,2)$, "a" $(13,14,15)$, and "M" $(0.9,0.91,0.92,0.93,0.94,0.95,0.96,0.97,0.98,0.99)$. Among the 152,460 tests, the parameters with the highest Jaccard index were $" c "=1.5, " g "=51, " f "=0.016, " j "=2, " a "=15$, and " $M "=0.95$. The above parameter optimization is compute intensive and a cluster computer with hundreds of central processing units is recommended. We applied the optimized parameters for all Drosophila species to generate sample-level de novo annotations. We then merged these sample-level annotations to species-level annotations for each species by StringTie (-merge). We optimized three parameters- “- $F$ " (minimum input transcript FPKM), “-T" (minimum input transcript transcripts per million), and "-g" (gap between transcripts to merge together) using the same optimization (Fig 5C). The optimal parameter combination was $F=0, T=10$, and $g=0$. We set minimum input transcript coverage ("-c") and minimum isoform fraction ("-f") as 1.5 
A

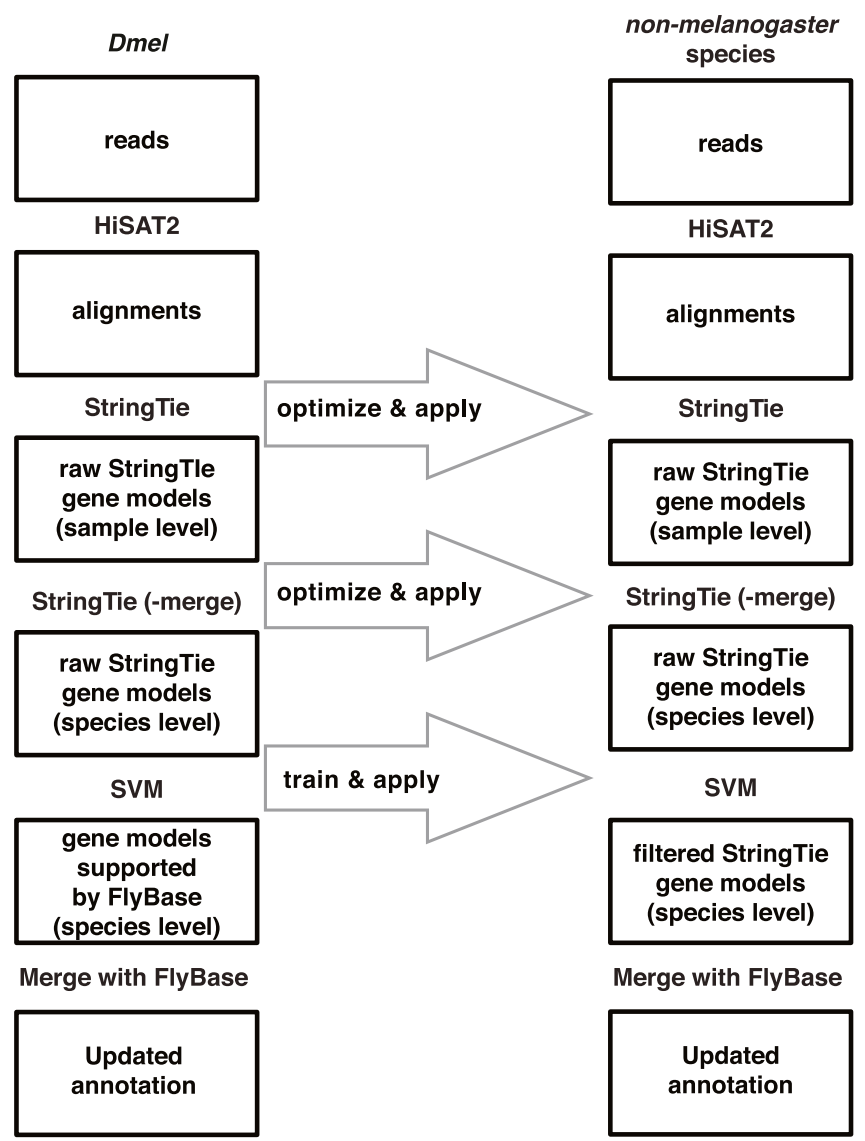

B

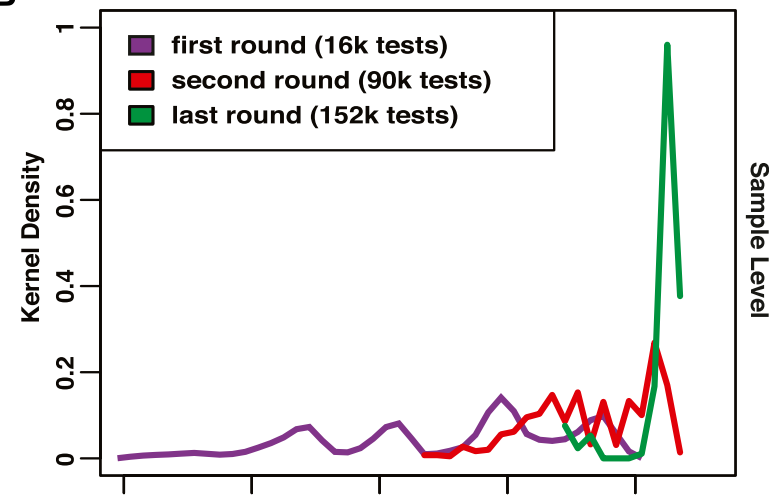

C

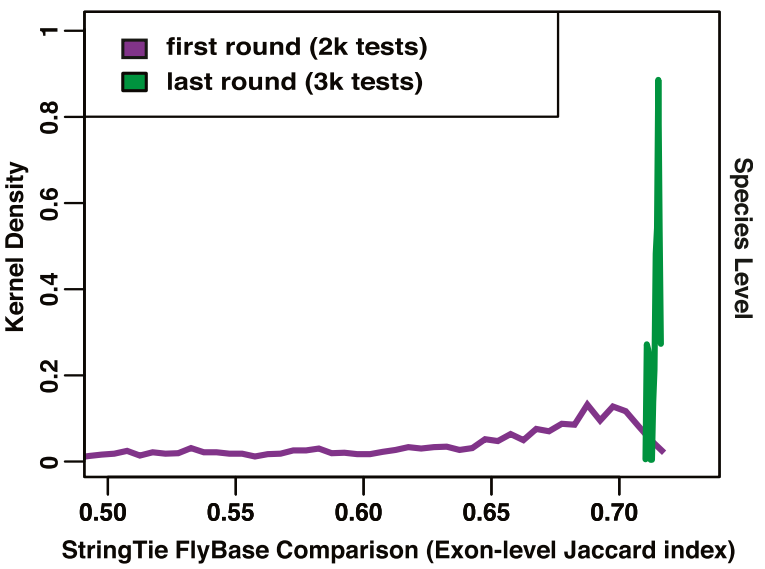

D

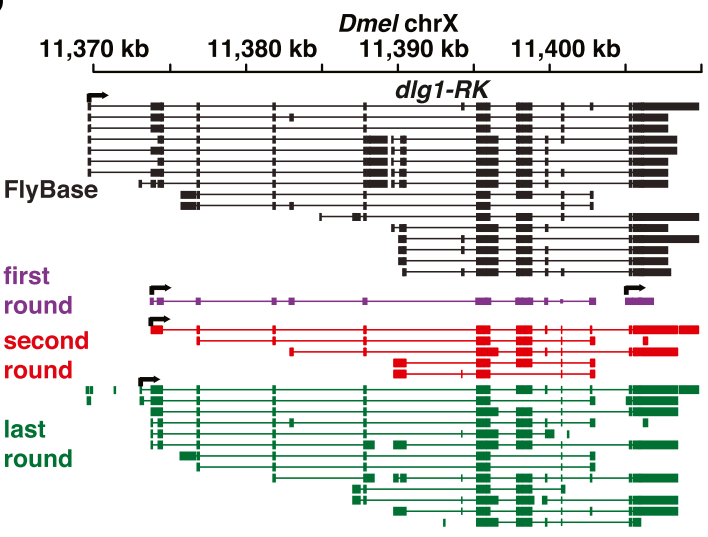

E

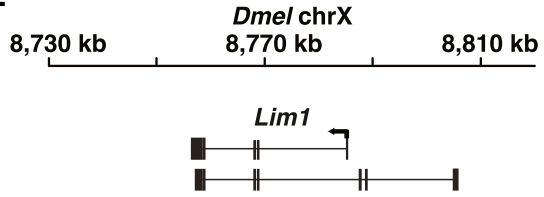

$\mathbf{F}$
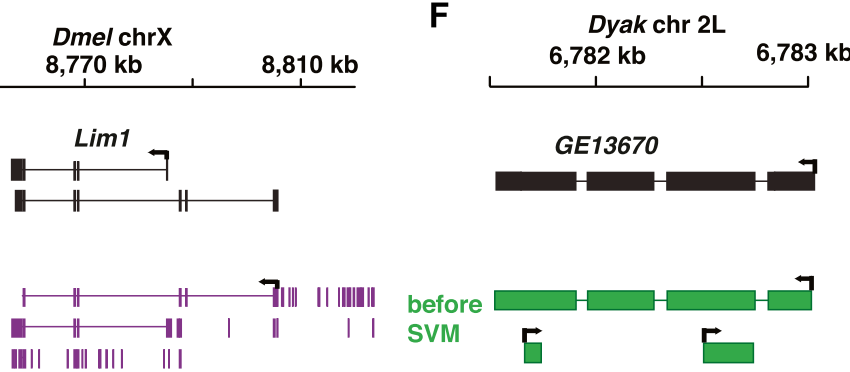

$\stackrel{\|}{+1} \quad \longrightarrow$

after SVM

Figure 5. Re-annotation and summaries.

(A) Re-annotation. From top to bottom, HiSAT2 was used to map all RNA-seq reads back to the appropriate genome. StringTie was used to generate sample-level annotations and merging annotations to the species-level. SVMs were used to train the recognition of FlyBase gene models based gene features (e.g., exon length, intron length, and isoforms. see the Materials and Methods section). In each species, the new annotation was eventually merged with the corresponding FlyBase annotation by gffcompare to create the new annotation. The same optimized parameters in Dmel (left column) were applied in each of the non-melanogaster species (right column). (B) In Dmel, we progressively converged sample-level annotations on the FlyBase annotation in three rounds of parameter optimization in StringTie. (C) Again in Dmel, we permuted and tested species-level annotations to maximize similarity to the FlyBase annotation during parameter optimization in StringTie (-merge). (B, C) The X-axis is the exon-level Jaccard index between StringTie and FlyBase annotation. The Y-axis is the distribution of the Jaccard index scores in kernel density. First (purple), second (red), and last (green) rounds of optimization are shown. (D) An example of gene model improvements when generating sample-level annotations. (E) An example of gene model improvements when generating species-level annotations. Note that most gene models with short, single, and intron-less transcripts were removed in the last round of parameter optimization. (F) An example of applying SVM in a non-melanogaster species. FlyBase gene model was shown in black, and Gene models before (light green) and after SVM (dark green) are shown. See Fig 2 for abbreviations and additional color coding information. 
Table 3. Sensitivity and precision of StringTie annotation after SVM filtration at different levels.

\begin{tabular}{|c|c|c|c|c|c|c|c|c|c|c|c|c|}
\hline & \multicolumn{2}{|c|}{ Base level } & \multicolumn{2}{|c|}{ Exon level } & \multicolumn{2}{|c|}{ Intron level } & \multicolumn{2}{|c|}{ Intron chain level } & \multicolumn{2}{|c|}{ Transcript level } & \multicolumn{2}{|c|}{ Gene level } \\
\hline & $s^{a}$ & $\mathbf{P}^{\mathbf{b}}$ & $S$ & P & S & P & S & P & S & $\mathbf{P}$ & S & P \\
\hline Dyak & 78.4 & 85.8 & 61.2 & 70.6 & 73.9 & 89.5 & 44.5 & 52.9 & 43.4 & 51.2 & 57.0 & 70.0 \\
\hline Dana & 78.1 & 85.5 & 59.7 & 70.6 & 71.9 & 89.7 & 45.3 & 53.9 & 44.2 & 52.7 & 55.8 & 70.5 \\
\hline Dpse & 74.8 & 84.9 & 55.0 & 66.9 & 68.6 & 87.6 & 37.4 & 48.1 & 36.6 & 47.4 & 51.5 & 68.8 \\
\hline Dper & 77.9 & 61.2 & 40.4 & 36.8 & 68.0 & 63.8 & 31.2 & 20.8 & 26.8 & 21.3 & 27.0 & 34.7 \\
\hline Dwil & 80.7 & 68.8 & 51.1 & 49.3 & 73.5 & 74.8 & 44.3 & 33.2 & 40.7 & 33.1 & 42.6 & 50.6 \\
\hline Dmoj & 79.1 & 83.1 & 59.4 & 65.3 & 73.1 & 86.2 & 44.2 & 46.2 & 43.1 & 45.9 & 54.7 & 67.9 \\
\hline Dvir & 78.4 & 84.6 & 58.6 & 66.7 & 72.1 & 87.9 & 43.4 & 48.4 & 41.7 & 47.6 & 54.2 & 67.3 \\
\hline Dgri & 79.2 & 59.1 & 44.5 & 37.6 & 74.3 & 66.2 & 37.3 & 23.4 & 31.0 & 22.8 & 31.2 & 38.8 \\
\hline $\mathrm{CV}^{\mathrm{C}}$ & 0.02 & 0.15 & 0.14 & 0.25 & 0.03 & 0.13 & 0.12 & 0.32 & 0.17 & 0.32 & 0.25 & 0.26 \\
\hline
\end{tabular}

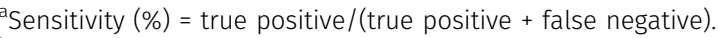

${ }^{\text {b}}$ Precision $(\%)=$ true positive/(true positive + false positive).

${ }^{c}$ Coefficient variation.

True positive: annotation identified in both StringTie and FlyBase.

False negative: annotation identified in FlyBase only.

False positive: annotation identified in StringTie only.

Values are obtained from gffCompare (with $-r$ reference annotation).

and as 0.016, respectively, to be consistent with previous StringTie settings. The effect of optimization on output is illustrated in Fig 5D. We applied the optimized parameters for all Drosophila species to generate draft species-level de novo annotations.

To identify which StringTie-predicted gene models were already annotated in FlyBase, we used the Jaccard index to calculate the similarity of gene structure between StringTie gene models and FlyBase. We used a cutoff (Jaccard index >0.6), to obtain $~ 10,000$ genes identified by both StringTie and FlyBase, and we deemed these genes as correctly predicted Dmel gene models of StringTie (Fig S1). However, we still observed an abundance of short single exon genes called by StringTie but not FlyBase (Fig 5E). To remove more of the StringTie unique calls, we used sequential and expressional features of genes to train a SVM (sklearn package v0.19.1 of Python v3.4.5) to recognize all the StringTie-predicted gene models with Jaccard index $>0.6$ with existing gene models in FlyBase. The features included isoform number, exon size, exon GC\%, intron size, intron GC\%, intron number (GT-AG intron type and other intron types, respectively), and median expression (DESeq2 normalized read counts) in 14 sexed tissues (except terminalia). For the SVM parameters, we tested different kernels (i.e., rbf, sigmoid), penalty parameter $C(0.001,0.01,0.1,1,10,100,1,000)$, and kernel coefficient $\gamma(0.001,0.01,0.1,1,10,100,1,000)$ values. The Receiver operating characteristic analyses indicated that the area under curve was the largest (0.97) at kernel $=\mathrm{rbf}$ and $\mathrm{C}=10$ and $y=0.1$. We also tested penalty parameter $C(0.001,0.01,0.1,1,10,100,1,000)$ under the linear kernel (kernel coefficient $y$ is not available for this kernel), the maximum area under curve we obtained is 0.95 , smaller than the optimal parameters. We applied the same SVM model with the optimized parameters to find qualified gene models in each species (Fig 5F). The sensitivity and precision of these annotations to FlyBase one were generated using gffCompare (with -r FlyBase annotation) and reported in Table 3. The drop of precision in Dper and Dgri for all levels (Table 3) is consistent with the fact that these two species had the poorest annotations among all (Fig $1 C$ and D).

To keep all StringTie gene candidates and FlyBase gene models in the updated annotation, we merged all qualified StringTie gene models with FlyBase annotation using gffCompare (v0.9.8) with option -r. In Dmel, we merged the StringTie gene candidates that were identified as correct prediction (i.e., Jaccard index $>0.6$ to FlyBase gene model) to the FlyBase annotation. If a StringTie transcript and a FlyBase transcript share the same structure for all introns on the same strand, we used the union of the gene structure of StringTie and FlyBase transcripts. After this step, the updated annotations were generated for each species. We used a universal ID format in the final updated annotations (e.g., YOgnYA12345). The format is Yang and Oliver (YO), gene (gn) or transcript (tr), species (Dmel (ME), Dyak (YA), Dana (AN), Dpse (PS), Dper (PE), Dwil (WI), $\operatorname{Dmoj}(\mathrm{MO})$, Dvir (VI), and Dgri (GR)), and a numerical identifier.

\section{Updated orthologs}

We used gene synteny, tissue-level expression, splicing conservation, and sequential similarity relative to Dmel to search for new orthologs (Fig 6A) with random gene pairs as a null model. To determine flanking gene synteny, we first identified the flanking 10 genes on each side of the target gene determined by the best reciprocal blast hits using blastp (Altschul et al, 1990) of all the known protein sequences in FlyBase (Fig 6B). To compare the expressional similarity, we plotted the normalized read counts of 14 tissues (all except terminalia) in the species gene relative to the Dmel gene (Fig 6C). When comparing the gene structure similarity of any gene pair between Dmel and non-melanogaster, we calculated the ratio of (unique intron number +1 ) between species gene and Dmel (Fig 6D). We used all known one-to-one orthologs between Dmel and non-melanogaster species to train an SVM 
A

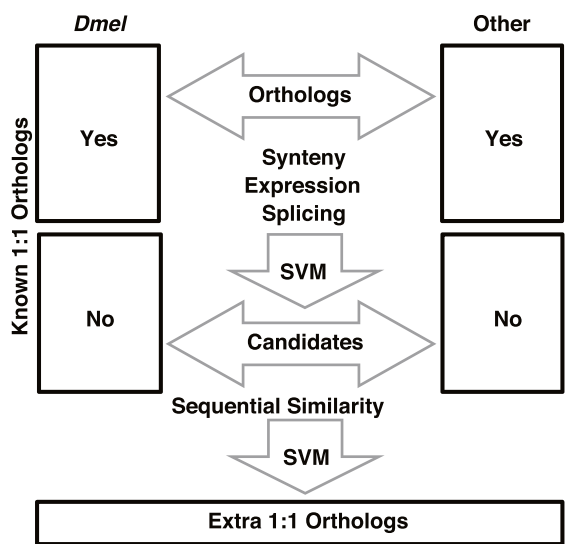

D

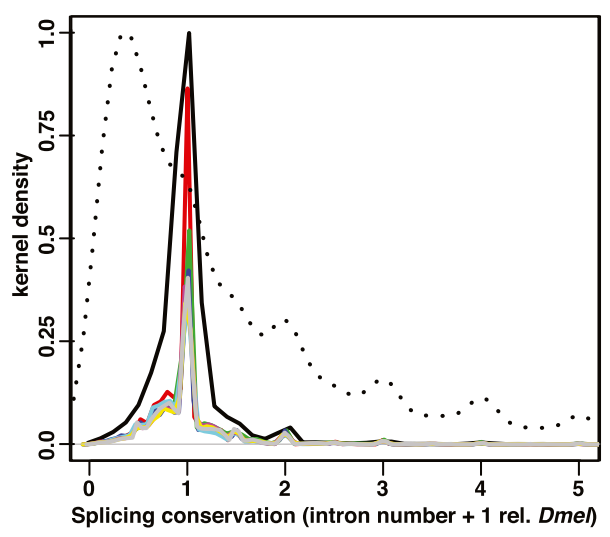

B

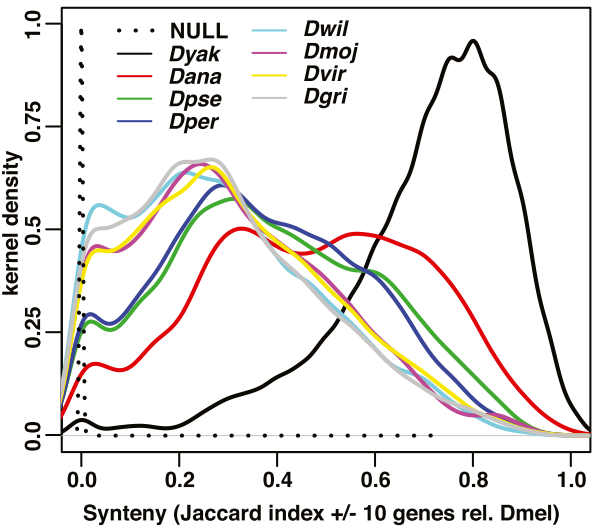

E

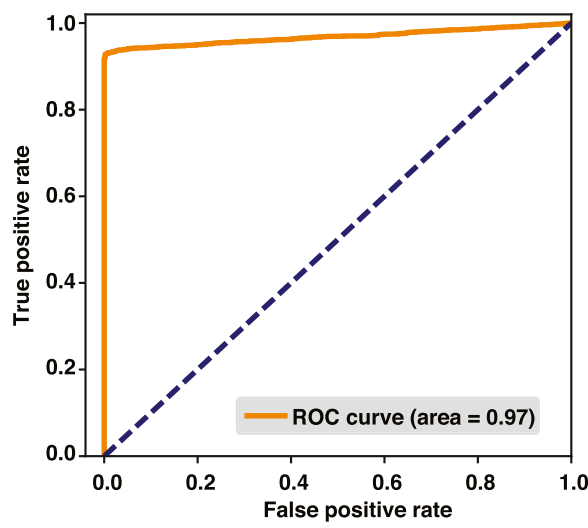

C

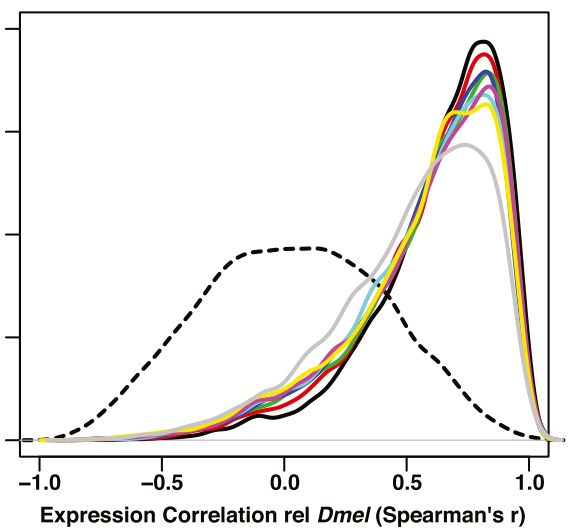

F

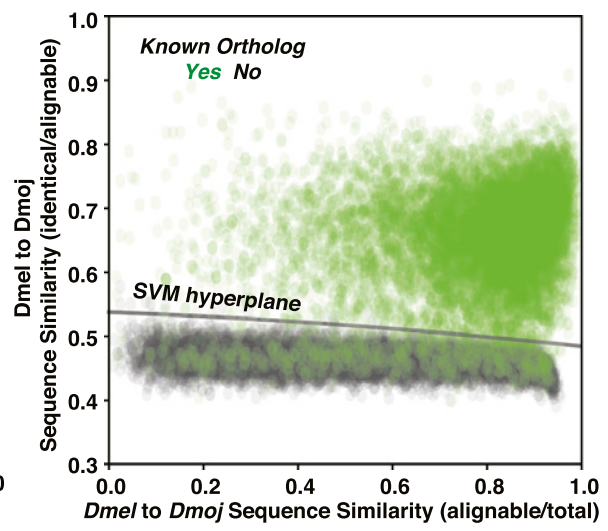

Figure 6. Ortholog identification and summaries.

(A) Pipeline to obtain extra 1:1 orthologs relative to Dmel. We used gene synteny, expression correlation among tissues, and exon structure similarity to train SVM models to recognize all known 1:1 orthologs. We used the same SVM model to generate more ortholog candidates among the genes that were not included in the current 1:1 ortholog dataset. Then we used sequence similarity (both alignable/total and identical/alignable) to finalize the extra 1:1 orthologs. (B) Distribution of orthologs in \pm 10 gene window surrounding the query gene relative to Dmel for each non-melanogaster species (in kernel density). Solid lines are distributions of previously reported 1:1 orthologs, and dotted line (NULL group) is the expected distribution based on random gene pairs (generated by python random package) between Dmel and Dyak (the other non-melanogaster species all generate identical distributions) in genome (the same for the following panels). (C) Distribution of expression similarity among orthologs (Spearman's r) relative to Dmel for each non-melanogaster species (in kernel density). For each ortholog, normalized read counts of 14 sexed tissues between Dmel and each non-melanogaster species were used to calculated correlation. (D) Distribution of intron number relative to Dmel for each non-melanogaster species. Intron number plus one was used to avoid an infinite value. The different distribution in Dyak compared with that of the other non-melanogaster species in (B) to (D) is presumably because of its evolutionary closeness to Dmel. (E, F) An example of SVM training of sequence similarity between Dmel and Dmoj. Receiver operating characteristic curve (E) and visualization of SVM training (F). Known orthologs are shown as green dots, whereas random gene pairs are shown as black dots. The SVM hyperplane is shown as a solid line.

model to recognize possible ortholog candidates based on the above three features. The optimal parameter combination was rbf kernel, $C=1,000$, and $y=0.001$. This procedure created a large number of potential orthologs. If there are multiple SVM predictions for one Dmel gene at this stage, we kept all these candidate orthologs. To be more conservative, we further filtered these ortholog candidates by using sequential similarity. We aligned the longest transcript of Dmel genes with that of non-melanogaster ortholog candidate by ClustalW (v2.1) with default parameters (Thompson et al, 1994). For each alignment, we calculated the ratio of alignable length to total length, and for the alignable region, we further calculated the ratio of identical length to alignable length. We used these two features to run SVM model (kernel $=r b f, C=1,000$ and $\gamma=0.01$ ) and selected the predicted orthologs (Fig $6 \mathrm{E}$ and $\mathrm{F}$ ). If there are multiple SVM predictions for one Dmel gene at this stage, we regarded them one-to-many orthologs and only used the one-to-one orthologs in the final results.

\section{Data Availability}

All data resources and their locations were listed in the reagent table (Table S1). Briefly, the following is available at GEO under GSE99574 and GSE80124 (see specific GSM\#s in Table S1): original RNA-seq reads (in fastq format); two demultiplexing versions, one based on HiSAT2 used here, and another generated by STAR; HTSeq raw read counts; gene-level DESeq2 normalized read counts; transcript-level expression from Salmon based on the updated 
annotation; bigWig tracks for each tissue, sex, and species; updated annotations for the nine Drosophila species (including Dmel, which includes novel isoforms but not novel genes because of it being used as the training dataset) in both gff and gtf format. PacBio Iso-seq CDNAs are provided in the SRA (SRP135764). We also provided the updated 1:1 ortholog table between Dmel and nonmelanogaster species. Junctions in bam format from HiSAT2 alignments of each sample (by SAMTools) are found at Zenodo (https://zenodo.org).

\section{Supplementary Information}

Supplementary Information is available at https://doi.org/10.26508/lsa. 201800156.

\section{Acknowledgements}

We are grateful to Ryan Dale, Justin Fear, Sharvani Mahadevaraju, Terence Murphy, Morgan Park, Harold Smith, Yijie Wang, and the Oliver lab for help and suggestions. The National Institute of Diabetes and Digestive and Kidney Diseases (NIDDK) Genomics core and the NIH Intramural Sequencing Center performed sequencing. This work utilized the computational resources of the NIH High-Performance Computing Biowulf cluster (http://hpc.nih.gov). This research was supported in part by the Intramural Research Program of the NIH, the NIDDK.

\section{Author Contributions}

H Yang: conceptualization, data curation, formal analysis, visualization, methodology, and writing-original draft, review, and editing.

M Jaime: resources and data curation.

M Polihronakis: resources and data curation.

K Kanegawa: resources and data curation.

T Markow: resources and data curation.

$K$ Kaneshiro: resources and data curation.

B Oliver: conceptualization, resources, data curation, formal analysis, supervision, funding acquisition, investigation, project administration, and writing-original draft, review, and editing.

$\mathrm{BO}$ and $\mathrm{HY}$ designed the experiment. MP, Kelvin Kanegawa, TM, and Kenneth Kaneshiro reared and maintained the flies. BO and $\mathrm{HY}$ dissected flies. HY and MJ performed RNA-seq library preparation. $\mathrm{HY}$ analysed the data. BO and HY wrote the manuscript. All authors read and approved the final manuscript.

\section{Conflict of Interest Statement}

The authors declare that they have no conflict of interest.

\section{References}

Adams MD, Celniker SE, Holt RA, Evans CA, Gocayne JD, Amanatides PG, Scherer SE, Li PW, Hoskins RA, Galle RF, et al (2000) The genome sequence of Drosophila melanogaster. Science 287: 2185-2195. doi:10.1126/science.287.5461.2185
Altschul SF, Gish W, Miller W, Myers EW, Lipman DJ (1990) Basic local alignment search tool. J Mol Biol 215: 403-410. doi:10.1006/ jmbi.1990.9999

Arbeitman MN, Furlong EE, Imam F, Johnson E, Null BH, Baker BS, Krasnow MA, Scott MP, Davis RW, White KP (2002) Gene expression during the life cycle of Drosophila melanogaster. Science 297: 2270-2275. doi:10.1126/ science.1072152

Ashburner M, Bergman CM (2005) Drosophila melanogaster: A case study of a model genomic sequence and its consequences. Genome Res 15: 1661-1667. doi:10.1101/gr.3726705

Bilder D, Irvine KD (2017) Taking stock of the Drosophila research ecosystem. Genetics 206: 1227-1236. doi:10.1534/genetics.117.202390

Brown JB, Boley N, Eisman R, May GE, Stoiber MH, Duff MO, Booth BW, Wen J, Park S, Suzuki AM, et al (2014) Diversity and dynamics of the Drosophila transcriptome. Nature 512: 393-399. doi:10.1038/ nature12962

Brown JB, Celniker SE (2015) Lessons from modENCODE. Annu Rev Genomics Hum Genet 16: 31-53. doi:10.1146/annurev-genom-090413-025448

Burtis KC, Baker BS (1989) Drosophila doublesex gene controls somatic sexual differentiation by producing alternatively spliced mRNAs encoding related sex-specific polypeptides. Cell 56: 997-1010. doi:10.1016/0092-8674(89)90633-8

Callaway E, Ledford H (2017) Medicine Nobel awarded for work on circadian clocks. Nature 550: 18. doi:10.1038/nature.2017.22736

Cech TR, Steitz JA (2014) The noncoding RNA revolution: Trashing old rules to forge new ones. Cell 157: 77-94. doi:10.1016/j.cell.2014.03.008

Celniker SE (2000) The Drosophila genome. Curr Opin Genet Dev 10: 612-616. doi:10.1016/s0959-437x(00)00140-4

Celniker SE, Rubin GM (2003) The Drosophila melanogaster genome. Annu Rev Genomics Hum Genet 4: 89-117. doi:10.1146/annurev. genom.4.070802.110323

Charlesworth D, Charlesworth B (2005) Sex chromosomes: Evolution of the weird and wonderful. Curr Biol 15: R129-R131. doi:10.1016/j.cub.2005.02.011

Chen ZX, Sturgill D, Qu J, Jiang H, Park S, Boley N, Suzuki AM, Fletcher AR, Plachetzki DC, FitzGerald PC, et al (2014) Comparative validation of the D. melanogaster modENCODE transcriptome annotation. Genome Res 24: 1209-1223. doi:10.1101/gr.159384.113

Chintapalli VR, Wang J, Dow JA (2007) Using FlyAtlas to identify better Drosophila melanogaster models of human disease. Nat Genet 39: 715-720. doi:10.1038/ng2049

Clough E, Jimenez E, Kim YA, Whitworth C, Neville MC, Hempel LU, Pavlou HJ, Chen ZX, Sturgill D, Dale RK, et al (2014) Sex- and tissue-specific functions of Drosophila doublesex transcription factor target genes. Dev Cell 31: 761-773. doi:10.1016/j.devcel.2014.11.021

Daines B, Wang H, Wang L, Li Y, Han Y, Emmert D, Gelbart W, Wang X, Li W, Gibbs R, et al (2011) The Drosophila melanogaster transcriptome by paired-end RNA sequencing. Genome Res 21: 315-324. doi:10.1101/ gr.107854.110

Drosophila 12 Genomes Consortium, Clark AG, Eisen MB, Smith DR, Bergman CM, Oliver B, Markow TA, Kaufman TC, Kellis M, Gelbart W, et al (2007) Evolution of genes and genomes on the Drosophila phylogeny. Nature 450: 203-218. doi:10.1038/nature06341

Drysdale R (2003) The Drosophila melanogaster genome sequencing and annotation projects: A status report. Brief Funct Genomic Proteomic 2: 128-134. doi:10.1093/bfgp/2.2.128

Edgar R, Domrachev M, Lash AE (2002) Gene expression omnibus: NCBI gene expression and hybridization array data repository. Nucleic Acids Res 30: 207-210. doi:10.1093/nar/30.1.207

Garber M, Grabherr MG, Guttman M, Trapnell C (2011) Computational methods for transcriptome annotation and quantification using RNA-seq. Nat Methods 8: 469-477. doi:10.1038/nmeth.1613 
Grabherr MG, Haas BJ, Yassour M, Levin JZ, Thompson DA, Amit I, Adiconis X, Fan L, Raychowdhury R, Zeng Q, et al (2011) Full-length transcriptome assembly from RNA-Seq data without a reference genome. Nat Biotechnol 29: 644-652. doi:10.1038/nbt.1883

Gramates LS, Marygold SJ, Santos GD, Urbano JM, Antonazzo G, Matthews BB, Rey AJ, Tabone CJ, Crosby MA, Emmert DB, et al (2017) FlyBase at 25: Looking to the future. Nucleic Acids Res 45: D663-D671. doi:10.1093/ nar/gkw1016

Graveley BR, Brooks AN, Carlson JW, Duff MO, Landolin JM, Yang L, Artieri CG, van Baren MJ, Boley N, Booth BW, et al (2011) The developmental transcriptome of Drosophila melanogaster. Nature 471: 473-479. doi:10.1038/nature09715

Haas BJ, Papanicolaou A, Yassour M, Grabherr M, Blood PD, Bowden J, Couger MB, Eccles D, Li B, Lieber M, et al (2013) De novo transcript sequence reconstruction from RNA-seq using the Trinity platform for reference generation and analysis. Nat Protoc 8: 1494-1512. doi:10.1038/ nprot.2013.084

Hoskins RA, Carlson JW, Wan KH, Park S, Mendez I, Galle SE, Booth BW, Pfeiffer BD, George RA, Svirskas R, et al (2015) The Release 6 reference sequence of the Drosophila melanogaster genome. Genome Res 25: 445-458. doi:10.1101/gr.185579.114

Hu TT, Eisen MB, Thornton KR, Andolfatto P (2013) A second-generation assembly of the Drosophila simulans genome provides new insights into patterns of lineage-specific divergence. Genome Res 23: 89-98. doi:10.1101/gr.141689.112

Jiang L, Schlesinger F, Davis CA, Zhang Y, Li R, Salit M, Gingeras TR, Oliver B (2011) Synthetic spike-in standards for RNA-seq experiments. Genome Res 21: 1543-1551. doi:10.1101/gr.121095.111

Kapustin Y, Souvorov A, Tatusova T, Lipman D (2008) Splign: Algorithms for computing spliced alignments with identification of paralogs. Biol Direct 3: 20. doi:10.1186/1745-6150-3-20

Kim D, Langmead B, Salzberg SL (2015) HISAT: A fast spliced aligner with low memory requirements. Nat Methods 12: 357-360. doi:10.1038/ nmeth.3317

Kuroda MI, Hilfiker A, Lucchesi JC (2016) Dosage compensation in Drosophilaa model for the coordinate regulation of transcription. Genetics 204: 435-450. doi:10.1534/genetics.115.185108

Leinonen R, Sugawara H, Shumway M; International Nucleotide Sequence Database Collaboration (2011) The sequence read archive. Nucleic Acids Res 39: D19-D21. doi:10.1093/nar/gkq1019

Leung W, Shaffer CD, Reed LK, Smith ST, Barshop W, Dirkes W, Dothager M, Lee $\mathrm{P}$, Wong J, Xiong D, et al (2015) Drosophila muller f elements maintain a distinct set of genomic properties over 40 million years of evolution. G3 (Bethesda) 5: 719-740. doi:10.1534/g3.114.015966

Lewis SE, Searle SM, Harris N, Gibson M, Lyer V, Richter J, Wiel C, Bayraktaroglu L, Birney E, Crosby MA, et al (2002) Apollo: A sequence annotation editor. Genome Biol 3: 1-82. doi:10.1186/gb-2002-3-12-research0082

Li H, Handsaker B, Wysoker A, Fennell T, Ruan J, Homer N, Marth G, Abecasis G, Durbin R; Genome Project Data Processing Subgroup (2009) The sequence alignment/map format and SAMtools. Bioinformatics 25 : 2078-2079. doi:10.1093/bioinformatics/btp352

Lu J, Fu Y, Kumar S, Shen Y, Zeng K, Xu A, Carthew R, Wu Cl (2008) Adaptive evolution of newly emerged micro-RNA genes in Drosophila. Mol Biol Evol 25: 929-938. doi:10.1093/molbev/msn040

Misra S, Crosby MA, Mungall CJ, Matthews BB, Campbell KS, Hradecky P, Huang Y, Kaminker JS, Millburn GH, Prochnik SE, et al (2002) Annotation of the Drosophila melanogaster euchromatic genome: A systematic review. Genome Biol 3: RESEARCH0083. doi:10.1186/gb-2002-3-12research0083
Parisi M, Nuttall R, Edwards P, Minor J, Naiman D, Lu J, Doctolero M, Vainer M, Chan C, Malley J, et al (2004) A survey of ovary-, testis-, and somabiased gene expression in Drosophila melanogaster adults. Genome Biol 5: R40. doi:10.1186/gb-2004-5-6-r40

Paris M, Villalta J, Eisen MB, Lott SE (2015) Sex bias and maternal contribution to gene expression divergence in Drosophila blastoderm embryos. PLOS Genet 11: e1005592. doi:10.1371/journal.pgen.1005592

Pertea M, Pertea GM, Antonescu CM, Chang TC, Mendell JT, Salzberg SL (2015) StringTie enables improved reconstruction of a transcriptome from RNA-seq reads. Nat Biotechnol 33: 290-295. doi:10.1038/nbt.3122

Pertea M, Kim D, Pertea GM, Leek JT, Salzberg SL (2016) Transcript-level expression analysis of RNA-seq experiments with HISAT, StringTie and Ballgown. Nat Protoc 11: 1650-1667. doi:10.1038/nprot.2016.095

Quinlan AR, Hall IM (2010) BEDTools: A flexible suite of utilities for comparing genomic features. Bioinformatics 26: 841-842. doi:10.1093/ bioinformatics / btq033

Richards S, Liu Y, Bettencourt BR, Hradecky P, Letovsky S, Nielsen R, Thornton K, Hubisz MJ, Chen R, Meisel RP, et al (2005) Comparative genome sequencing of Drosophila pseudoobscura: Chromosomal, gene, and cis-element evolution. Genome Res 15: 1-18. doi:10.1101/gr.3059305

Robertson G, Schein J, Chiu R, Corbett R, Field M, Jackman SD, Mungall K, Lee S, Okada HM, Qian JQ, et al (2010) De novo assembly and analysis of RNA-seq data. Nat Methods 7: 909-912. doi:10.1038/nmeth.1517

Sturgill D, Malone JH, Sun X, Smith HE, Rabinow L, Samson ML, Oliver B (2013) Design of RNA splicing analysis null models for post hoc filtering of Drosophila head RNA-Seq data with the splicing analysis kit (Spanki). BMC Bioinformatics 14: 320. doi:10.1186/1471-2105-14-320

Souvorov A, Kapustin Y, Kiryutin B, Chetvernin V, Tatusova T, Lipman D (2010) Gnomon-NCBI Eukaryotic Gene Prediction Tool. https://www.ncbi. $\mathrm{nlm}$.nih.gov/core/assets/genome/files/Gnomon-description.pdf.

Tautz D (2009) Polycistronic peptide coding genes in eukaryotes: How widespread are they? Brief Funct Genomic Proteomic 8: 68-74. doi:10.1093/bfgp/eln054

Thompson JD, Higgins DG, Gibson TJ (1994) CLUSTAL W: Improving the sensitivity of progressive multiple sequence alignment through sequence weighting, position-specific gap penalties and weight matrix choice. Nucleic Acids Res 22: 4673-4680. doi:10.1093/nar/ 22.22.4673

Vieira FG, Sanchez-Gracia A, Rozas I (2007) Comparative genomic analysis of the odorant-binding protein family in 12 Drosophila genomes: Purifying selection and birth-and-death evolution. Genome Biol 8: R235. doi:10.1186/gb-2007-8-11-r235

Vijay N, Poelstra JW, Kunstner A, Wolf JB (2013) Challenges and strategies in transcriptome assembly and differential gene expression quantification. A comprehensive in silico assessment of RNA-seq experiments. Mol Ecol 22: 620-634. doi:10.1111/mec.12014

Yi W, Zarkower D (1999) Similarity of DNA binding and transcriptional regulation by Caenorhabditis elegans MAB-3 and Drosophila melanogaster DSX suggests conservation of sex determining mechanisms. Development 126: 873-881.

Zook JM, Samarov D, McDaniel J, Sen SK, Salit M (2012) Synthetic spike-in standards improve run-specific systematic error analysis for DNA and RNA sequencing. PLoS One 7: e41356. doi:10.1371/journal.pone.0041356

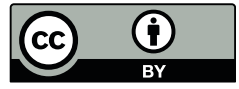

License: This article is available under a Creative Commons License (Attribution 4.0 International, as described at https://creativecommons.org/ licenses/by/4.0/). 